\title{
EL PROCESO DE INFILTRACIÓN EN LOS BADLANDS DEL ESTE DE LA PENÍNSULA IBÉRICA. AVANCES Y RETOS
}

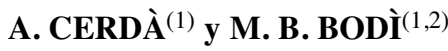 \\ (1) Departament de Geografia \\ Universitat de València. Blasco Ibáñez, 28, 46010 València
}

(2) GEA (Grupo de Edafología Ambiental). Departamento de Agroquímica y Medio Ambiente Universidad Miguel Hernández, Avenida de la Universidad s/n, 03202 Elche, Alicante

Correo electrónico de contacto: artemio.cerda@uv.es

RESUMEN. Los badlands son laboratorios geomorfológicos en miniatura que han permitido conocer más y mejor los procesos y las formas terrestres. La infiltración es un proceso clave en el ciclo hidrológico ya que gestiona el volumen de las aguas superficiales y por lo tanto los procesos erosivos. Estudiar el proceso de infiltración en los badlands permite conocer mejor ambos-el proceso de infiltración y los badlands-y los resultados obtenidos se pueden extrapolar a otros espacios en los que las altas pérdidas de suelo y agua se deben reducir. En este artículo se presenta una revisión del estado de la cuestión respecto a la infiltración en los badlands a partir de experimentos desarrollados en las dos últimas décadas en los badlands del este peninsular. El uso de lluvia simulada y de infiltrómetros de cilindro ha aportado información relevante que ha permitido conocer el efecto del roquedo, de la estacionalidad mediterránea, del tiempo y de la posición geomorfológica sobre el proceso de infiltración, e indirectamente sobre la generación de escorrentía y pérdida de suelo.

A pesar de la falta de cubierta vegetal, y del escaso desarrollo edáfico, el proceso de infiltración es altamente complejo en los badlands debido a que en ellos se generan macroporos a partir de las grietas que favorecen la infiltración del agua y con ello la formación de tubificaciones (piping). A falta de vegetación, es el roquedo a través de la morfología superficial (grietas) el factor dominante en los procesos de infiltración en los badlands. También la posición topográfica es clave para entender los procesos geomorfológicos en zonas de badlands ya que los pediments se comportan con simples transmisores de agua y sedimentos que llegan de las laderas debido a su reducida infiltrabilidad y falta de grietas. Las grietas explican la elevada estacionalidad en la capacidad de infiltración del regolito. Así, las condiciones de los badlands propician que el proceso de infiltración-altamente variable estacional, temporal y espacialmente- sea el proceso clave en los procesos geomorfológicos. 
ABSTRACT. Badlands are geomorphological miniature laboratories that contributed to the landforms and geomorphological processes studies. Infiltration is a key process in the hydrological cycle because it manages the volume of surface water and thus the erosive processes. Studying the process of infiltration into badlands gain insight on both, the process of infiltration and on badland geomorphology, and the results can be extrapolated to other areas where the high losses of soil and water must be controlled. This paper presents a review of the research regarding the infiltration in the badlands from experiments developed in the last two decades in the badlands of eastern Iberian Peninsula. The use of rainfall simulation experiments and cylinder infiltrometers measurements has provided relevant information to determine the effects of parent material, seasonality, time and geomorphological position on the process of infiltration, and then on the runoff generation and soil loss. Despite the lack of vegetation cover, and low soil development, the process of infiltration is highly complex in the badland surfaces because they generate macroporos from cracks that favor water infiltration and thus the piping developement. In the absence of vegetation, is the parent material through the surface morphology (cracks) the dominant factor in the processes of infiltration. Topographical position is key to understand the geomorphological processes in areas of badlands because the pediments behave as simple transmitters of water and sediment coming from the slopes because of its small infiltrabilidad and lack of cracks. Cracks also explain why the seasonality in the infiltration capacity of the regolith is high. Thus, the infiltration process in badlands is characterised by a highly seasonal, temporal and spatial variability which is key on the geomorphological processes behaviour.

Palabras clave: Badlands, Infiltration, Cracks, Piping, Eastern Spain.

Key words: Badlands, Infiltración, Grietas, Tubificaciones, Este de España.

Enviado el 15 de septiembre de 2008 Aceptado el 20 de noviembre de 2008

\section{Introducción. Los badlands}

Los badlands son superficies sin o con escasa cubierta vegetal en las que los procesos erosivos son muy activos como lo demuestran los valles o cárcavas en forma de $\mathrm{V}$ (Campbell, 1974; Bryan y Yair, 1982a). Estos ambientes están presentes en todo el Planeta, pero son las zonas áridas y semiáridas donde son más abundantes (Yair et al., 1980; Alexander, 1982; Imeson et al., 1982; Laronne, 1982; Scoging, 1982; Alexander et al., 1994; Gutiérrez Elorza et al., 1997). El clima mediterráneo, caracterizado por la sequía estival y la irregularidad también propicia el desarrollo de badlands (Vittorine, 1977; Yair et al., 1982; Imeson et al., 1988; Harvey y Calvo, 1991; Cerdà y Navarro, 1997; Gallart et al., 2002). Por ello, los badlands se encuentran en las zonas climáticas cercanas a los desiertos, si bien no están ausentes en zonas húmedas (Bryan, 1974; Campbell, 
1982; Hodges y Bryan, 1982; Howard, 1994; Regüés y Gallart, 1996; Nadal Romero et al., 2007). En aquellos ecosistemas donde el aporte hídrico es suficiente para que la cubierta vegetal esté presente, pero escaso para que la vegetación cubra el suelo de forma permanente y eficiente, es donde los badlands tienen más posibilidades de prosperar (Campbell, 1989). En esas circunstancias de reducida cubierta vegetal cualquier alteración fruto de cambios climáticos, tectónicos, en la dinámica fluvial o la acción del hombre, favorece el desarrollo de las cárcavas, la pérdida del suelo y la desaparición de la vegetación protectora, lo que se traduce en la presencia de la roca madre parcialmente alterada en superficie (regolito o regolita) y de un paisaje en el que dominan las geoformas sobre la vegetación: los badlands (Wells y Gutiérrez, 1982).

Además del clima semiárido que favorece la presencia de los badlands, es necesario que la roca madre sea altamente erosionable, y para ello las rocas friables y blandas como las margas, son las más propicias (Bryan y Yair, 1982b; Clotet et al., 1987). No obstante, también se forman badlands sobre depósitos cuaternarios no consolidados y sobre otros materiales como las arcillas. Las condiciones climáticas y litológicas permiten encontrar badlands en cualquier continente, desde el Desierto de Judea en los territorios ocupados por Israel, al departamento de Tarija en Bolivia o en Nuevo México en EE.UU. (Fig. 1). También, el sudeste de la península ibérica, y en general en toda la vertiente mediterránea, presentan paisajes acarcavados o abarrancados sobre litologías blandas (Solé, 2007). Esta constante se hace más evidente en las zonas más áridas y es allí donde las incisiones de los cauces son más evidentes debido a la actividad geotectónica. Buenos ejemplos de ello son los badlands de las provincias de Almería (Desierto de Tabernas), Granada (Guadix), Murcia (Vera), Alicante (riu Montnegre), Valencia (Requena), pero también los espacios situados más al norte como la Bardenas Reales (Fig. 2a y 2b).

La presencia de "malas tierras" en el sudeste de la península ibérica es debido a la combinación de un clima semiárido con fuertes variaciones estacionales en la precipitación y la temperatura, además de sequías prolongadas, con roquedos blandos y friables que favorecen la remoción del material (López Bermúdez, 1992). También es de destacar aquí la elevada intensidad de las lluvias extraordinarias que puede alcanzar y superar los 200 $\mathrm{mm} \mathrm{día}{ }^{-1}$ (incluso $800 \mathrm{~mm} \mathrm{día}^{-1}$ ) y que puntualmente puede desarrollar cárcavas que sólo son funcionales durante esos eventos extraordinarios pero que quedan ya en el paisaje para siempre (Pérez Cueva, 1994). Además, durante las lluvias extraordinarias se generan movimientos en masa que elimina la capa superficial del suelo y deja el roquedo en superficie, lo que favorece la formación de cárcavas, de badlands. Pero, además del control climático debemos hacer mención especial a la activa neotectónica del sudeste peninsular que propicia la continua incisión de los cauces, la zapa basal de las laderas y la erosión remontante que hace que la roca madre quede sin la cubierta protectora del suelo y que los procesos de erosión sean muy activos (Wise et al., 1982; Calvo et al., 1991a).

En el mediterráneo, donde la actividad humana es ancestral, los badlands están íntimamente relacionados con el uso agrícola, ganadero, minero o forestal. Por una parte, y de forma indirecta, la eliminación de la vegetación por el pastoreo o los incendios recurrentes puede desencadenar la aceleración de la erosión de los suelos, y la formación de 

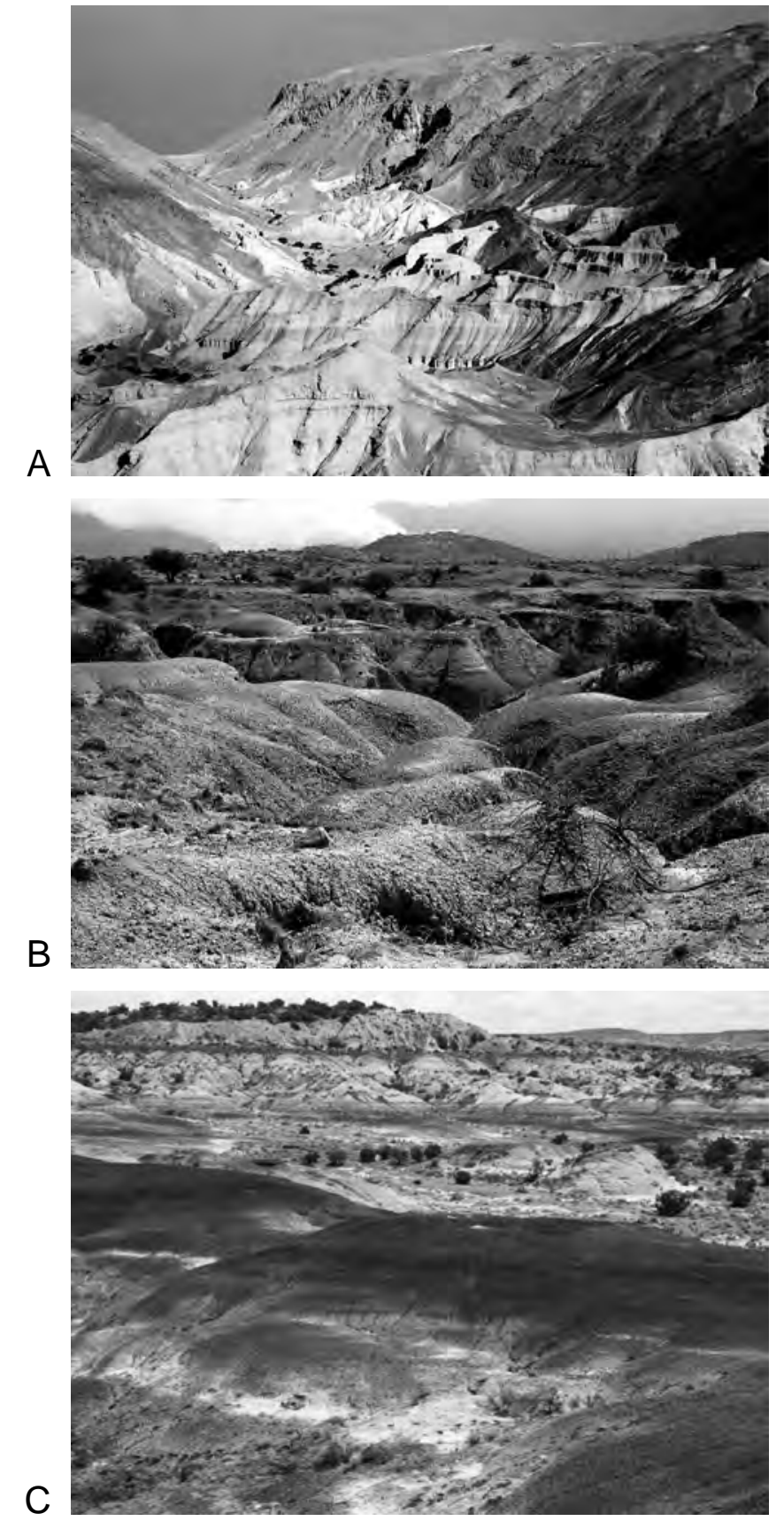

Figura 1. Badlands en distintas partes del mundo. A. Desierto de Judea en los territorios ocupados (Israel); B. Departamento de Tarija (Bolivia); y C. Nordoeste de Nuevo México (EE.UU.) 


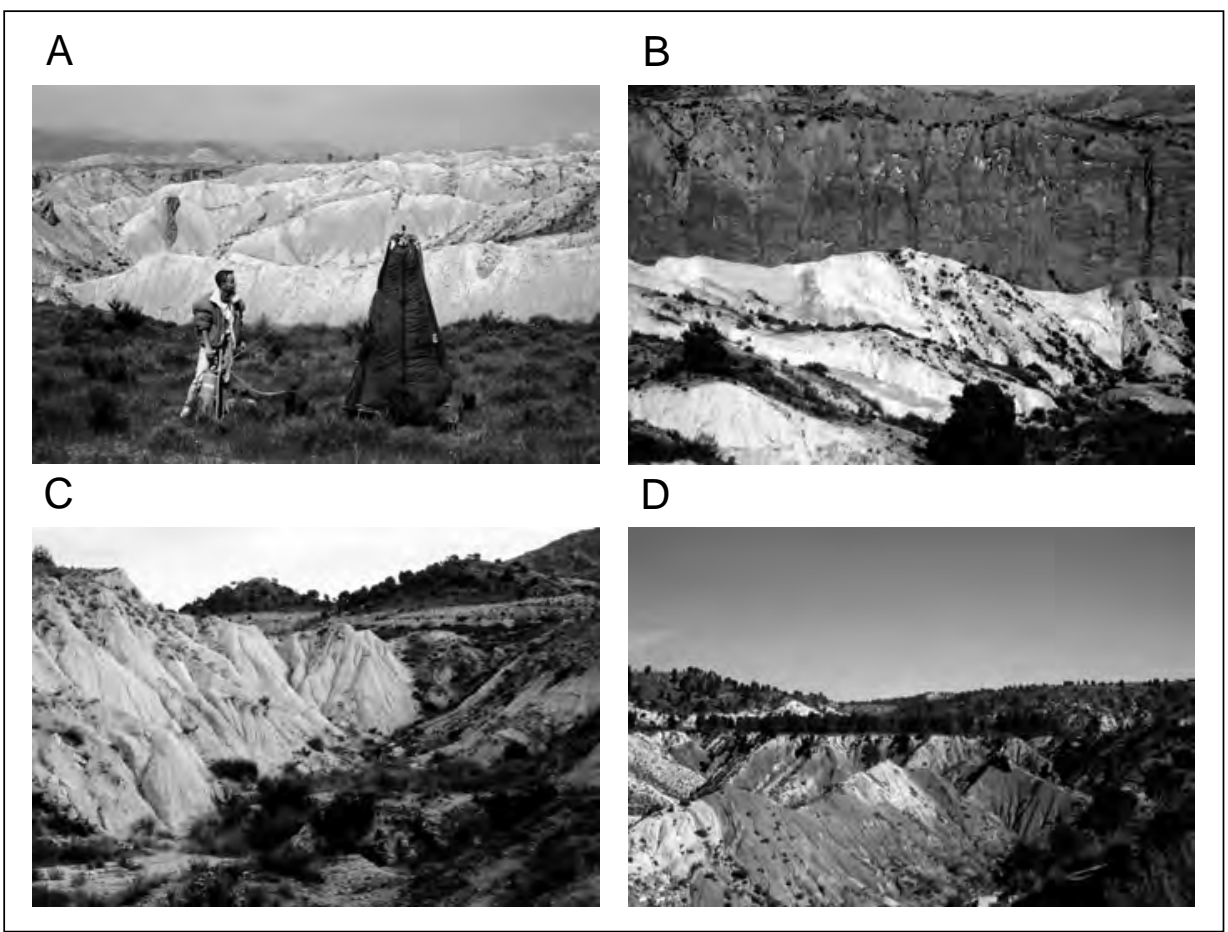

Figura 2a. Badlands en el sudeste peninsular. A. Desierto de Tabernas, Almería; B. Riu Monnegre, Alicante; C. Petrer, Alicante; y D. Requena, Valencia

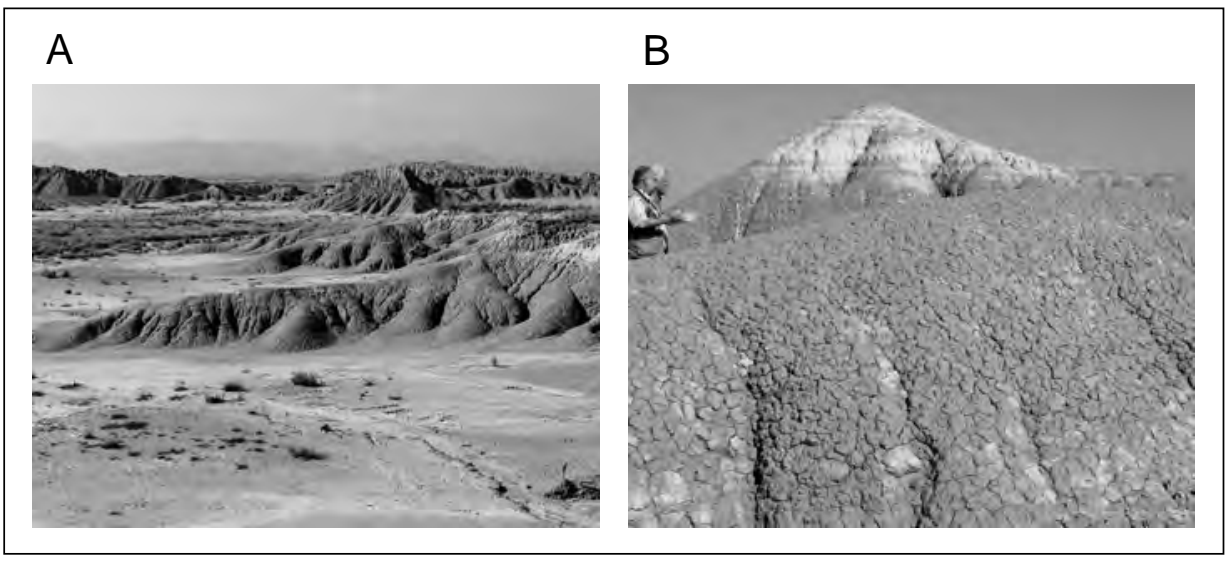

Figura 2b. A. Vista de los badlands de las Bardenas Reales. A. Conjunto de laderas y pediments.

$B$. Detalle de la ladera de badlands con un intenso agrietamiento, encostramiento

y con la presencia de reguereos. En esta última fotografía Aaron Yair y Rorke Bryan ejercen amablemente de escala. Ambas fotos fueron tomadas por Albert Solé 
cárcavas. Por otra, el cultivo puede desestabilizar las laderas e iniciarse un proceso de erosión que desmantela de forma paulatina los suelos (Rodríguez Aizpeolea, 1992). La formación de badlands por la acción antópica puede ser incluso más directa si es posible con la minería (derrubios), desmontes para campos de cultivo (nivelación) y con los taludes de carretera (Nicolau, 2002; Cerdà, 2007). De hecho, en la actualidad existen extensas superficies de badlands en las vías de comunicación del sudeste peninsular como consecuencia de los desmontes y taludes de carretera que no han sido revegetados con éxito (Fig. 3). También ocurre lo mismo con los derrubios de minas (Nicolau, 2002), los espacios en los que se construyen infraestructuras (Cerdà, 2007), en los desmontes de las nuevas plantaciones de viñas (Meyer y Martínez Casasnovas, 1999) o cítricos y en zonas recientemente quemadas (Cerdà y Doerr, 2007). Es cierto que estos ambientes generados por el hombre nunca han sido considerados como badlands por la literatura científica, ya que no están en espacios naturales. Sin embargo, tampoco son espacios completamente naturales aquellos estudiados como badlands ya que en ellos también la mano del hombre -como en todo el paisaje mediterráneo- ha sido decisiva en su evolución reciente. De hecho, el origen antrópico de los badlands del sudeste peninsular, por causa directa (arado) o indirecta (cambio climático) es bien conocido (Wise et al., 1982; Rodríguez Aizpeolea, 1992). Además, hay que reconocer que los estudios desarrollados en los badlands son aplicables a estas zonas degradadas en las que la vegetación es esca-

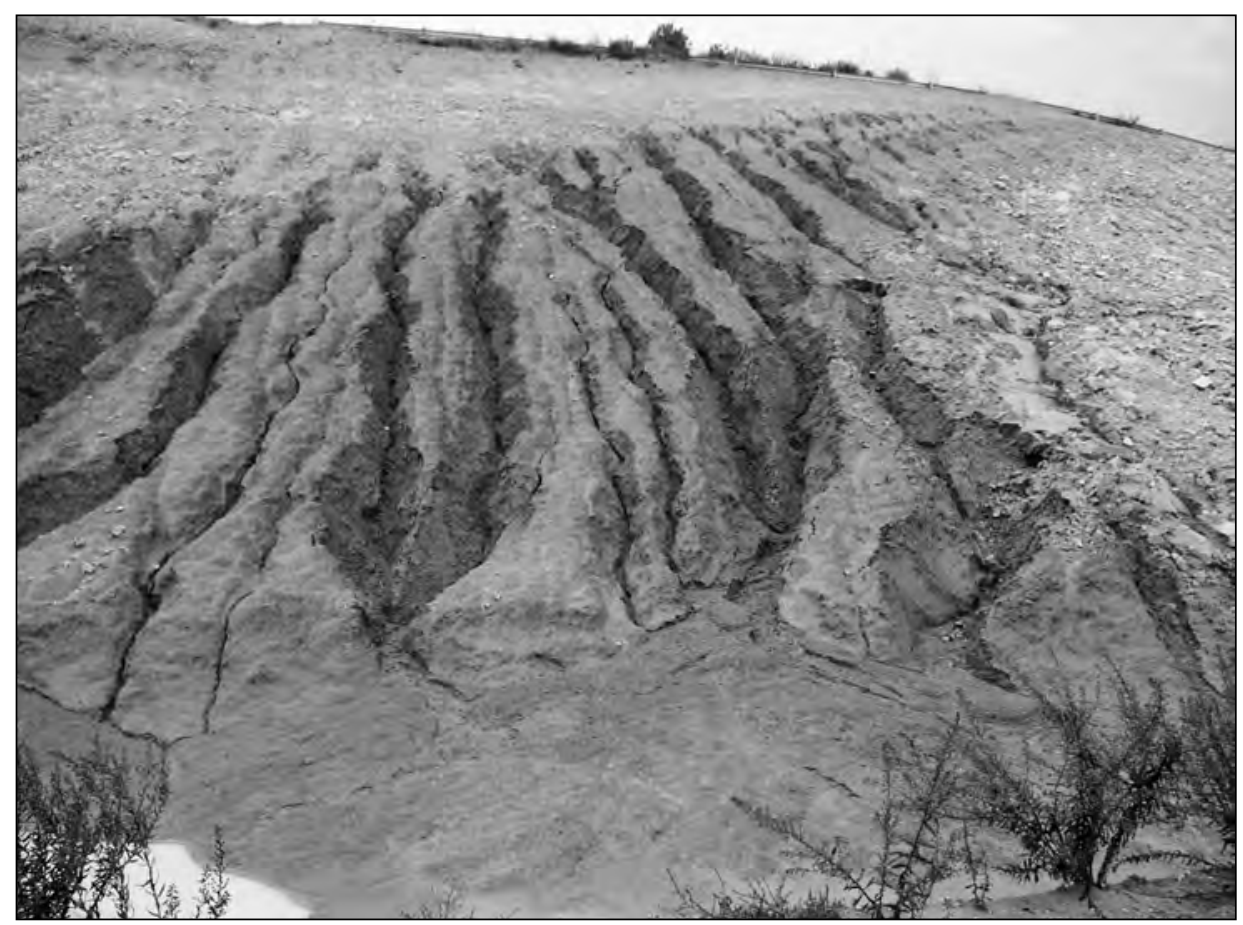

Figura 3. ¿Taludes de carretera o badlands? Talud de carretera en el sur de la provincia de Valencia 
sa y los caudales y la pérdida de suelo son extraordinarios. Por lo tanto, los estudios realizados en lo badlands, además de contribuir al conocimiento básico sobre hidrología, geomorfología, botánica, ecología o edafología, tiene una aplicación práctica sobre estos nuevos espacios (taludes, derrubios...) que nuestra sociedad genera con tanta facilidad y rapidez.

Los badlands, despreciados por los agricultores y denostados por las autoridades públicas, son en cambio magníficos paisajes de gran interés científico ya que en ellos se presentan una gran variedad de formas y procesos geomorfológicos en un reducido espacio. Movimientos en masa, formación de regueros y cárcavas, incisión acelerada, costras físicas, químicas y biológicas, grietas y polígonos pueden ser estudiados en detalle. De hecho, los badlands son entendidos como laboratorios geomorfológicos en los que en reducidos espacios podemos analizar los cambios espaciales y temporales de distintos procesos y formas terrestres. Esos estudios pueden servir por una parte para conocer la dinámica geomorfológica de los badlands y por otra para buscar soluciones para espacios en los que las pérdidas de agua y sedimentos no son deseables como los apuntados anteriormente: derrubios de minas, desmontes para obras civiles o agrícolas, campos de cultivo y taludes de carretera (Fig. 3). Hay que recordar que la contribución de los badlands a la escorrentía y sobretodo a la producción de sedimentos es relevante en la producción de sedimentos a escala de cuenca de drenaje (Bryan et al., 1984; Regüés et al., 1998; Beguería, 2005). Pero para entender los procesos que actúan en los badlands es clave conocer el proceso de infiltración ya que de él depende la gestión de la lluvia y su transformación en escorrentía superficial o en agua del suelo (Dunin, 1976). Conocer la capacidad de infiltración de los suelos de los badlands también aportará información sobre las posibilidades de recuperación de la cubierta vegetal, al depender la germinación y la viabilidad de las plántulas del agua infiltrada. Y como en tantos otros aspectos, conocer el proceso de infiltración en los badlands permitirá conocer su funcionamiento en otros ámbitos al ser laboratorios en miniatura.

\section{La infiltración en los badlands. Procesos, métodos y factores}

La literatura científica sobre hidrología siempre entendió que la infiltración del agua en los suelos (regolito) de los badlands era escasa o incluso nula (Campbell, 1987). En esa afirmación se han basado muchos estudios sobre los procesos hidrológicos y erosivos en los badlands, aunque no es del todo cierta (Cerdà, 1993a). Es bien sabido que los badlands generan grandes caudales, y se ha dado por bueno que la escorrentía se genera como consecuencia de la superación de la capacidad de infiltración de los suelos por la lluvia según fue explicado por Horton (1933), y es por ello súbita (Leopold et al., 1964; Schumm et al., 1986; Imeson, 1983). De hecho, Horton desarrolló su trabajo en base a espacios semiáridos, en los que los badlands no son extraños. Sin embargo, algunos resultados apuntan a que no siempre los badlands se comportan como una superficie impermeable (Cerdà, 1995b), y en ello tiene mucho que ver la presencia de grietas que permiten el flujo en macroporos mientras están abiertas y de tubificaciones o 
"piping”, que es otra característica muy extendida de los badlands (López Bermúdez y Torcal, 1986; Gutiérrez Elorza et al., 1997; Cerdà, 1999a). Además, los cambios en el regolito en los badlands presentan una evolución estacional muy marcada que afectará a los procesos de infiltración (Cerdà y Payà, 1995). En este sentido se deben consultar los trabajos de David Regüés donde se detalla la dinámica en la morfología de los badlands (Regüés et al., 1992; 1993).

La forma más sencilla de medir la infiltración de los suelos es encharcándolos y cuantificar recurrentemente la altura de ese encharcamiento (Hills, 1970). El uso de infiltrómetros de doble anillo o de grandes espacios inundados ha sido la forma más habitual de medir la infiltración. Esto es sencillo en campos de cultivo pero no en laderas que alcanzan incluso los $45^{\circ}$ de pendiente en algunas cárcavas. Así, se optó por el uso de un infiltrómetro de cilindro de tan solo $7 \mathrm{~cm}$ de diámetro, se eliminó el segundo anillo para simplificar el trabajo de campo, aumentar el número de mediciones, y de esa forma realizar mediciones en cualquier posición topográfica. Se mantenía el infiltrómetro encharcado durante una hora y se recargaba cada minuto tras la oportuna medida del nivel del encharcamiento (Cerdà, 1993b; 1995a). Las mediciones con infiltrómetro de cilindro en distintas zonas del este peninsular informaron de que bajo suelos encharcados los badlands presenta tasas de infiltración altamente variables, y superiores a lo esperado en valores medios, aunque siguen siempe menores a cualquier uso del suelo excepto las zonas impermeabilizadas por el hombre. La tabla 1 muestra la tasa de infiltración final estable medida con infiltrómetro de cilindro. Destaca la elevada variabilidad como demuestra el que la desviación estándar sea superior a la media. No se encontraron diferencias significativas entre las cuatro zonas de estudio, y por lo tanto la capacidad de infiltración no mostró diferencias entre zonas de margas y arcillas bajo encharcamiento. Hay que apuntar que las tasas de infiltración final estable medias fueron superiores a los 100 $\mathrm{mm} \mathrm{h}^{-1}$, y que puntualmente algunos suelos superaron los $500 \mathrm{~mm}$ año ${ }^{-1}$. En las cuatro zonas de estudio no se superan los $500 \mathrm{~mm}$ año-1 de precipitación, y algunos casos como Petrer se alcanzan con dificultad los $300 \mathrm{~mm}_{\text {año-1 }}{ }^{-1}$ (Pérez Cueva, 1994). Por lo tanto, la medición de la capacidad de infiltración mediante encharcamiento no aportaba un valor real de la tasa a la que actúa el proceso, ya que es evidente la formación de escorrentías abundantes en las zonas de badlands con precipitaciones que superen los $20 \mathrm{~mm} \mathrm{~h}^{-1}$, y de caudales extraordinarios cuando se superan los $50 \mathrm{~mm} \mathrm{~h}^{-1}$ (Berndtsson et al., 1985; Calvo et al., 1991b; Cerdà, 1996a; Cantón et al., 2001).

Las mediciones realizadas con infiltrómetro de cilindro se realizaron sobre una superficie de $38,78 \mathrm{~cm}^{2}$ y siempre mediante encharcamiento artificial de entre 1 y $5 \mathrm{~cm}$. La infiltración en las laderas de los badlands se produce durante las lluvias y estas son variables en intensidad y volumen, y no se producen encharcamientos superiores a 2-3 mm debido a que los charcos son rápidamente drenados por un relieve que suele ser abrupto y con superficies que están bien organizadas por el drenaje (Sirvent et al., 1997; Solé et al., 1997; Cantón et al., 2004). Por ello, se procedió a realizar las mismas mediciones, siempre durante el verano, pero bajo lluvia simulada y en una superficie 2 órdenes de magnitud mayor $\left(2375,77 \mathrm{~cm}^{2}\right.$ ) bajo lluvia simulada (Cerdà, 1993b; 1997; 1999a). Este ha sido un método utilizado por distintos investigadores en distintos espacios (Bryan, 
Tabla 1. Tasa de infiltración final estable, ifc (o conductividad hidraúlica, Ks) para 80 suelos en badlands situados en margas del Cretácico (Petrer y Monnegre) y arcillas del Keuper (Toris y Requena) medida mediante infiltrómetro de cilindro sencillo de $7 \mathrm{~cm}$ de diámetro. Estas muestras fueron tomadas el verano de 1991

\begin{tabular}{|c|r|r|r|r|}
\hline Zonas & Monnegre & Petrer & Anna & Toris \\
\hline $\mathbf{1}$ & 251,62 & 89,28 & 494,42 & 356,02 \\
$\mathbf{2}$ & 17,59 & 61,73 & 73,35 & 199,75 \\
$\mathbf{3}$ & 193,52 & 288,04 & 49,79 & 320,39 \\
$\mathbf{4}$ & 50,81 & 353,21 & 218,76 & 88,89 \\
$\mathbf{5}$ & 73,99 & 404,54 & 503,11 & 230,61 \\
$\mathbf{6}$ & 14,34 & 12,25 & 56,91 & 22,81 \\
$\mathbf{7}$ & 11,40 & 130,09 & 118,53 & 16,38 \\
$\mathbf{8}$ & 75,61 & 12,52 & 22,70 & 103,32 \\
$\mathbf{9}$ & 84,98 & 270,30 & 108,41 & 89,20 \\
$\mathbf{1 0}$ & 104,88 & 7,35 & 427,54 & 155,11 \\
$\mathbf{1 1}$ & 98,48 & 27,51 & 22,60 & 86,50 \\
$\mathbf{1 2}$ & 579,72 & 25,60 & 46,95 & 65,99 \\
$\mathbf{1 3}$ & 605,22 & 32,85 & 58,00 & 470,95 \\
$\mathbf{1 4}$ & 866,40 & 141,63 & 38,94 & 15,21 \\
$\mathbf{1 5}$ & 97,85 & 35,75 & 154,84 & 28,11 \\
$\mathbf{1 6}$ & 88,77 & 49,19 & 112,98 & 47,74 \\
$\mathbf{1 7}$ & 9,74 & 23,43 & 107,43 & 772,41 \\
$\mathbf{1 8}$ & 11,37 & 39,71 & 32,40 & 330,44 \\
$\mathbf{1 9}$ & 147,97 & 28,13 & 213,99 & 164,64 \\
$\mathbf{2 0}$ & 123,74 & 274,32 & 155,12 & 703,90 \\
\hline Media & $\mathbf{1 7 5 , 4 0}$ & $\mathbf{1 3 4 , 9 9}$ & $\mathbf{1 5 0 , 8 4}$ & $\mathbf{2 1 3 , 4 2}$ \\
\hline Std & $\mathbf{2 3 3 , 4 7}$ & $\mathbf{1 4 0 , 8 6}$ & $\mathbf{1 5 1 , 7 2}$ & $\mathbf{2 2 1 , 0 3}$ \\
\hline & & & & \\
\end{tabular}

1981; Agassi et al., 1994; Meyer et al., 1994; Arnáez et al., 2007), pero especialmente en badlands donde la escorrentía superficial es abundante (Bryan et al., 1984; Alexander y Calvo, 1990). Con anterioridad se utilizó exclusivamente escorrentía artificial para conocer la dinámica hidromorfológica de los badlands (Schumm y Lusby, 1963; Schumm, 1964). De hecho, la aplicación de lluvia simulada fue un avance substancial y algunos simuladores adaptaron instrumentos para su uso en esas difíciles condiciones topográficas y climáticas (Wilcox et al., 1986; Cerdà et al., 1997; Cerdà, 1999b). Otros métodos como el número de curva han sido menos aplicado (Wood, 1987). 
La intensidad de la lluvia seleccionada fue de $55 \mathrm{~mm} \mathrm{~h}^{-1}$, y se mantuvo durante 30 minutos con el fin de reproducir chaparrones intensos de un periodo de recurrencia inferior a 5 años en las de estudio (Elías Castillo y Ruiz Beltrán, 1979). Las escorrentías y las lluvias se median instantáneamente (cada minuto) con lo que se podía conocer la tasa de infiltración instantánea al substraer la primera de la segunda. La tasa de infiltración fue calculada siguiendo la ecuación de Horton (1940) tanto para el infiltrómetro de cilindro como para la lluvia simulada,

$$
f=f_{c}+(f o-f c) e^{-\alpha} \quad F=f c t-\left(f_{o}-f_{c}\right) / \alpha\left(e^{-\alpha} t-1\right)
$$

Donde: $f=$ tasa de infiltración instantánea, $F=$ tasa de infiltración acumulada, $f c=$ tasa de infiltración final estable, $f o=$ tasa de infiltración inicial $(t=0), \alpha=$ constante (se identifica con la forma de la curva de infiltración)

Los resultados obtenidos muestran diferencias significativas entre las zonas de margas (Monnegre y Petrer) y las de arcillas (Anna y Toris), lo que demuestra un claro efecto del roquedo que no se hacía evidente con el infiltrómetro de cilindro. Además, las tasas de infiltración medidas bajo lluvia simulada fueron mucho menores a las medidas por los infiltrómetros de cilindro. Estos resultados informan de una clara influencia del método de medición sobre el resultado. La Fig. 4 muestra como la tasa de infiltración final estable medida con infiltrómetro de cilindro (ifc) no es distinta entre zonas y roquedos, mientras que si lo es cuando es medida con lluvia simulada $(f c)$.

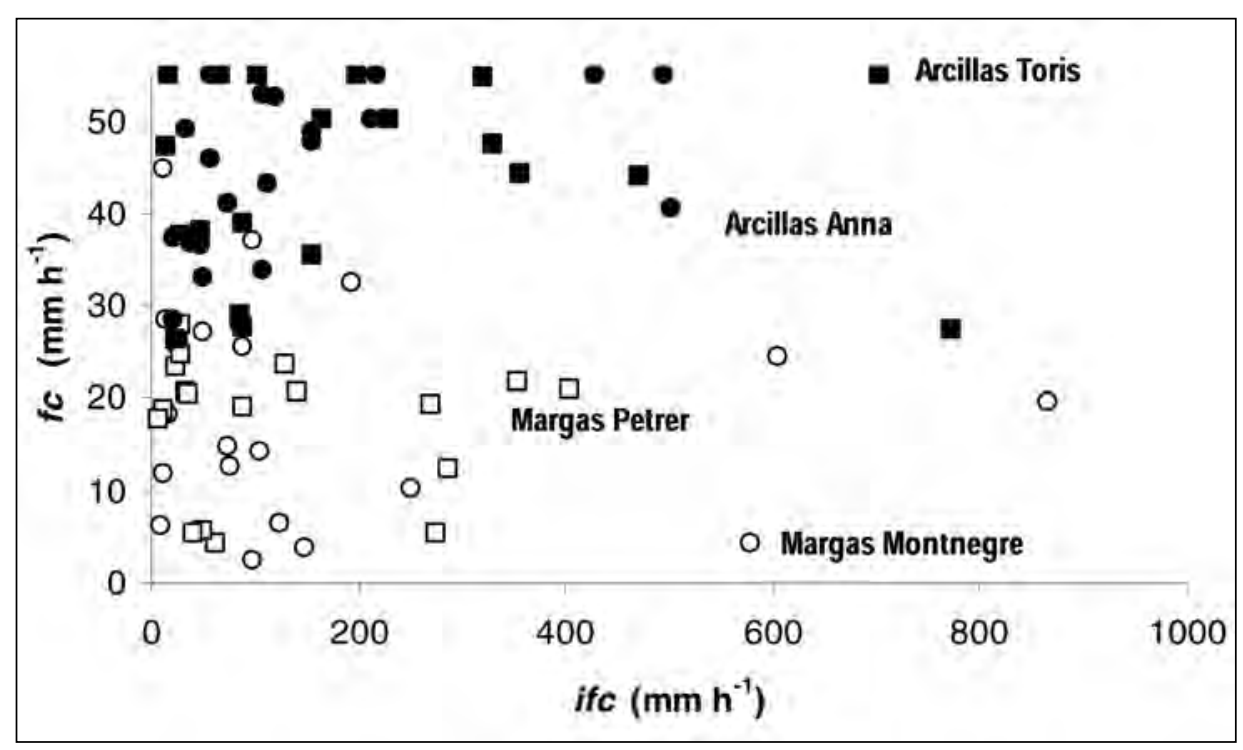

Figura 4. Tasa de infiltración final estable medida con infiltrómetro de cilindro (ifc) y bajo lluvia simulada a $55 \mathrm{~mm} \mathrm{~h}^{-1}$ de intensidad $(f c)$ 
Las diferencias en magnitud entre if $c$ y $f c$ son distintas entre las margas y las arcillas. En concreto, la tasa de infiltración final estable medida en las margas es 8 veces mayor que en las arcillas cuando se mide con infiltrómetro de cilindro, y 4 veces mayor si la medición es realizada mediante lluvia simulada a $55 \mathrm{~mm} \mathrm{~h}^{-1}$ de intensidad durante 30 minutos. También la variabilidad espacial es distinta cuando se utilizan distintos métodos. El coeficiente de variación es superior a 100 en las cuatro zonas de estudio cuando se mide con infiltrómetro de cilindro y se reduce drásticamente cuando la medición se realiza con lluvia simulada. Estas diferencias se deben a que: (i) la máxima tasa de infiltración medida con lluvia simulada es la de la intensidad de la lluvia $\left(55 \mathrm{~mm} \mathrm{~h}^{-1}\right)$ mientras que la máxima tasa medida con infiltrómetro de cilindro es hipotéticamente infinita, si bien nunca hemos medido tasas mayores a $2000 \mathrm{~mm} \mathrm{~h}^{-1}$. La otra razón, la de mayor interés geomorfológico, reside en que el proceso de infiltración es altamente variable en las superficies de badlands debido a la morfología superficial con abundantes grietas (Scoging, 1982) y a la formación en superficie de las cabeceras de las tubificaciones o piping (Harvey, 1982; López Bermúdez y Romero Díaz, 1989). La importancia de las tubificaciones fue puesto de relieve por Jones (1981) para zonas húmedas, pero los badlands se han revelado como uno de los espacios en los que la escorrentía subsuperficial concentrada es más eficiente y evidente hasta el punto de aparecer en el título de la publicación emblemática de la geomorfología de badlands: "Badland Geomorphology and Piping” (Bryan y Yair, 1982a). La investigación geomorfológica ha permitido conocer la relevancia de este proceso que fue olvidado durante décadas seguramente porque es muy difícil de cuantificar. Incluso en campos de cultivo, las tubificaciones se han mostrado como un elemento relevante (García Ruiz et al., 1997), y tendríamos que extender a otros ambientes el estudio de las tubificaciones como un proceso relevante en la hidrogeomorfología de espacios semiaáridos.

La tabla 1 muestra como la variabilidad espacial es muy alta cuando las mediciones se realizan mediante encharcamiento. Esto es debido a que cuando el infiltrómetro es colocado en la zona entre grietas la tasa de infiltración se reduce a valores extremadamente bajos $\left(<50 \mathrm{~mm} \mathrm{~h}^{-1}\right)$, cuando hay alguna grieta se alcanzan valores elevados (> $50 \mathrm{~mm} \mathrm{~h}^{-1}$ ) y en ocasiones hemos encontrado tasas de infiltración ascendentes (mayores al final del experimento que al principio) debido a que las grietas internas se han conectado con la superficie donde se encuentra instalado el infiltrómetro (ver ejemplos A, B y C para la Fig. 5 y la Fig. 6 con ejemplos de tubificaciones y la Fig. 7A con imágenes obtenidas a partir de la lluvia simulada). A los pocos minutos de la humectación (por lluvia o encharcamiento) las zonas entre grietas se convierten en impermeables debido al encostramiento superficial típico de los badlands y a la presencia de arcillas expandibles (Römkens et al., 1990), de ahí que $f c$ (tasa de infiltración final estable) sea extremaamente baja cuando no hay grietas.

En las mediciones con lluvia simulada, el impacto de la gota y la salpicadura favorecen la homogeneización de la superficie. También el hinchamiento de las arcillas contribuye al cierre de grietas y por lo tanto a la impermeabilización de la superficie del regolito. Algunos roquedos propician el mantenimiento de grietas durante varios minutos después de iniciada la escorrentía, la cual termina por colmatar las grietas o bien por 


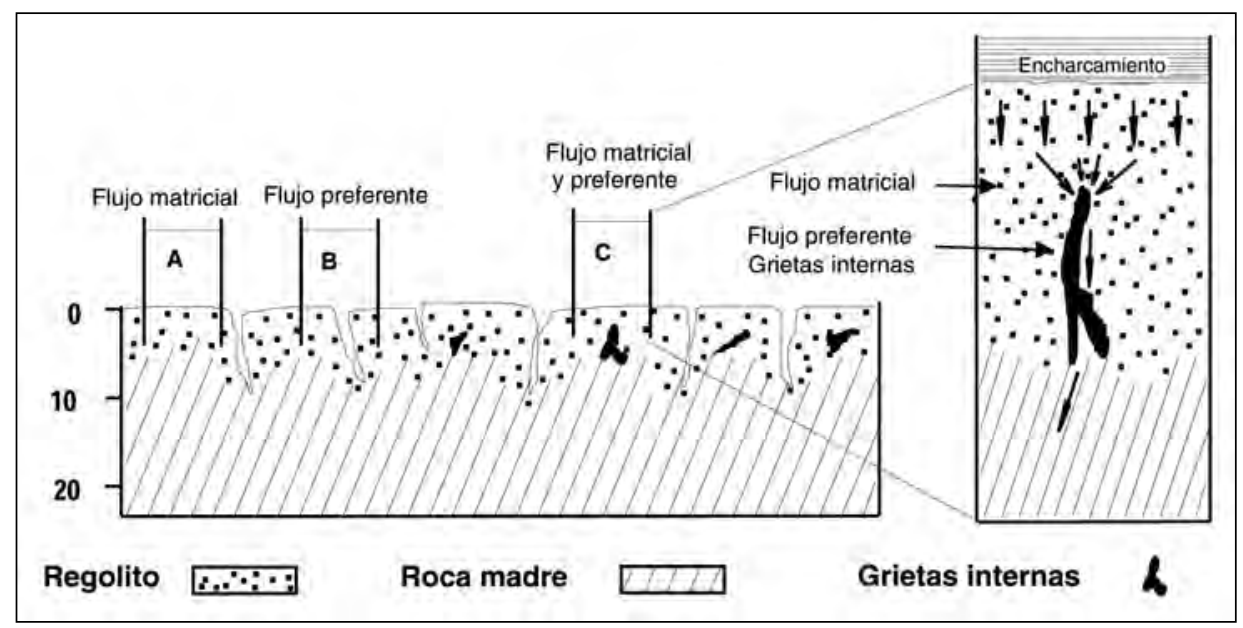

Figura 5. Reconstrucción de las mediciones con encharcamiento en badland

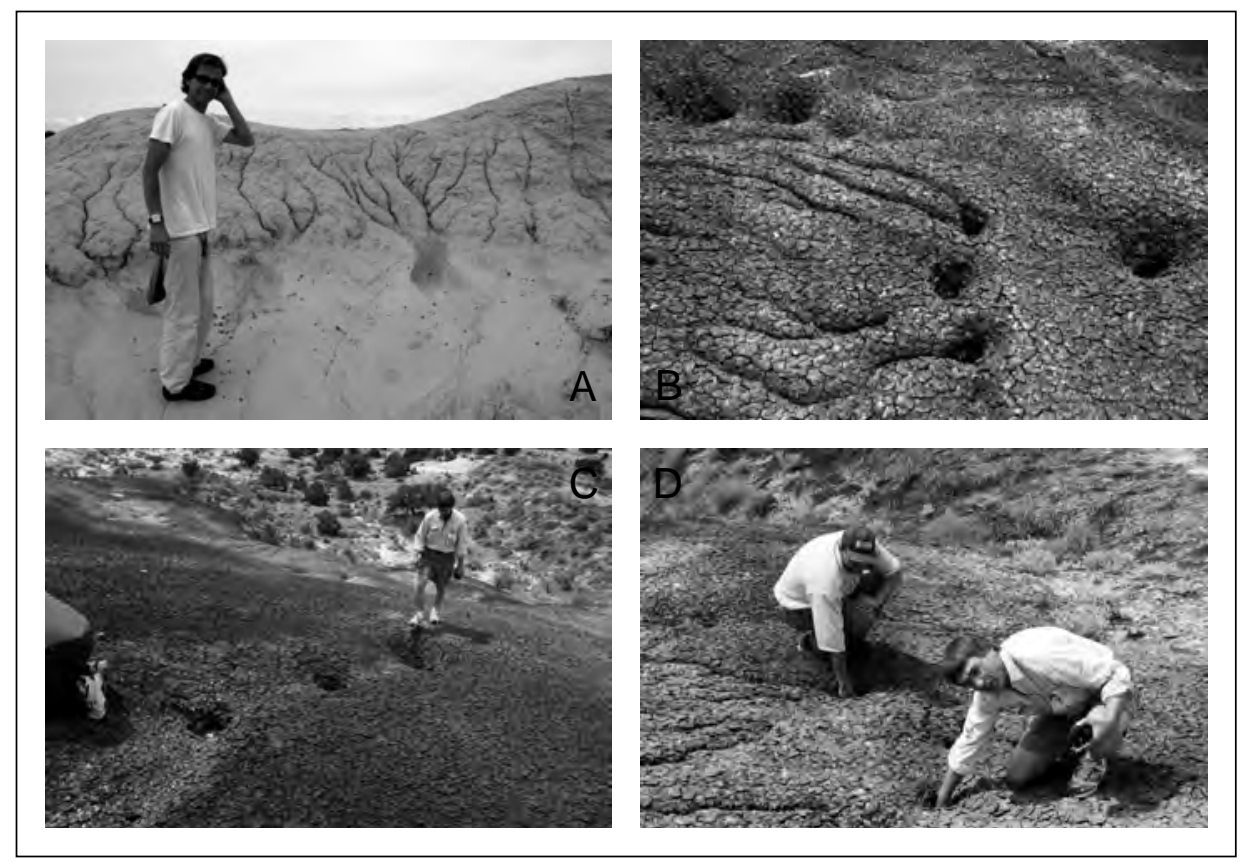

Figura 6. Vista de badlands en los que las escorrentías superficiales son dominantes (A) y otros en los que esas escorrentías se transforman en flujo subsuperficial concentrado en forma de tubificaciones o piping $(B, C, y D)$. La presencia de montmorillonita es decisiva en estos badlands situados en el oeste de New México (EE.UU.) 


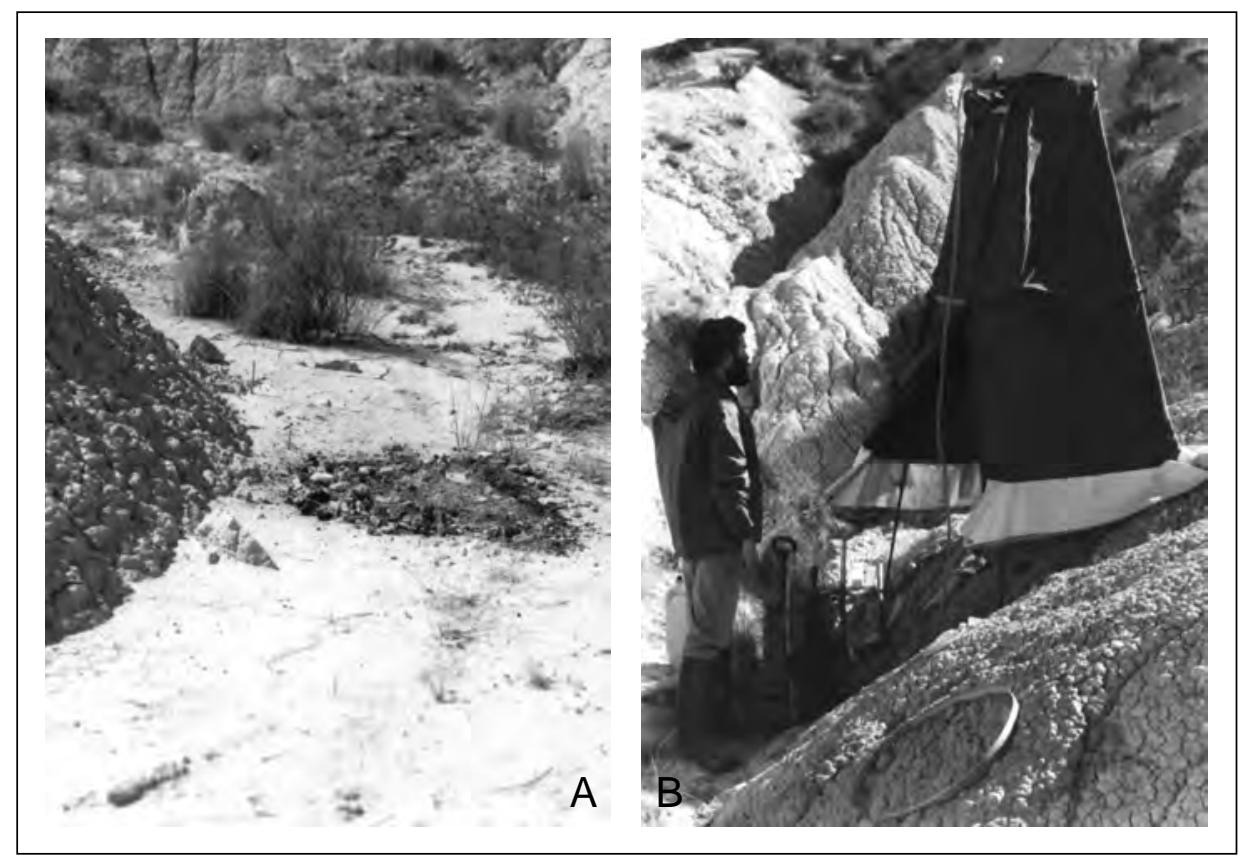

Figura 7. A) Vista del contacto entre la ladera y el pediment del badland. En la foto se aprecia una zona del pediment en la que se incorporó materia orgánica y semillas (entre otras estrategias aplicadas) que nunca consiguieron ralentizar el proceso de erosión. B) Vista del trabajo de campo con el simulador de lluvia en una ladera, con una parcela en primer plano

favorecer el flujo subsuperficial (Fig. 8). En las arcillas del Keuper las grietas son más profundas y persistentes por lo que no es extraño encontrar parcelas en badlands donde se infiltre toda la lluvia de chaparrones de intensidades elevadas (Tabla 2). Sin embargo, lo habitual es que se cuantifiquen coeficientes de escorrentía elevados, debido a las bajas tasas de infiltración, especialmente en zonas de margas. Hay que apuntar que la presencia de Montmorillonita en las margas siempre da lugar a escorrentías inferiores y a mayores tasas de infiltración debido a la persistencia de la grietas.

Estos experimentos realizados con lluvia simulada y las mediciones con infiltrómetro de cilindro en cuatro zonas de estudio sobre badlands en las provincias de Alicante y Valencia permitieron concluir que: (i) la capacidad de infiltración de los suelos (regolito) en los badlands es baja, la más bajas dentro de los ecosistemas mediterráneo (Cerdà, 1995b), pero con alta variabilidad espacial dependiente de la presencia de grietas (y tubificaciones); (ii) el uso del infiltrómetro de cilindro no es aconsejable en un espacio en el que el impacto de la gota y la salpicadura son relevantes en la respuesta erosiva e hidrológica de los suelos, aunque su uso ayuda a entender la importancia de los macroporos en la generación de escorrentía y la erosión subsuperficial; (iii) las tubificaciones (piping) se pueden iniciar allí donde las grietas superficiales se conectan con un macro- 


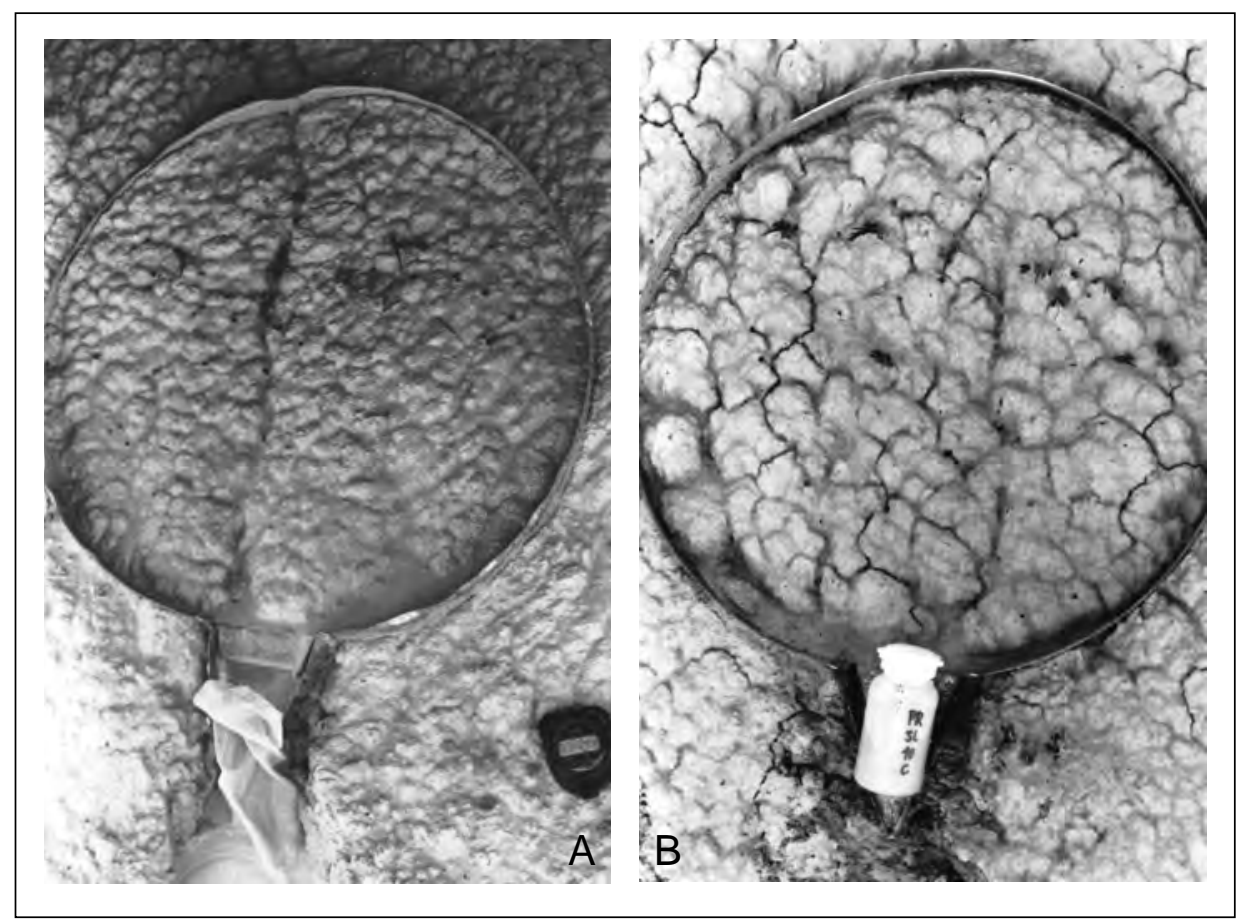

Figura 8. Vista de dos parcelas después de un chaparrón de 27,5 mm durante 30 minutos en Petrer, Alicante. La figura A muestra como el suelo ha quedado completamente sellado, mientras que en la B aún se muestran grietas abiertas. Esta es la clave para entender el proceso de infiltración en los badlands y desmentir la creencia generalizada de que el proceso de infiltración es insignificante en estos espacios

poro del regolito, o incluso allí donde el encharcamiento permite la conexión de la escorrentía superficial con los macroporos; y (iv) el uso de la lluvia simulada aporta información clave para conocer la dinámica hidrogeomorfológica de los badlands, por lo que es el método más adecuado para el estudio de los badlands.

Los retos de la investigación en el futuro residen en el estudio de la influencia del tamaño de la parcela ya que se sospecha que la falta de vegetación, de suelos porosos y la elevada eficiencia en el transporte (regueros y cárcavas) pueden provocar un aumento de la escorrentía al aumentar el área de estudio, lo que no suele ocurrir en otros espacios donde se produce un aumento de la infiltración al aumentar la superficie estudiada. Además aquí se debe contar con la escorrentía concentrada subsuperficial. Otra línea de investigación a desarrollar es la dirigida a la restauración de los suelos y el control de la erosión, que será de gran utilidad en su aplicación a taludes de carretera, zonas degradadas, derrubios de minas o incluso campos de cultivo. Estas actuaciones deben de aplicarse allí donde sea estrictamente necesario ya que los paisajes de los badlands son un patrimonio geomorfológico a proteger. 
Tabla 2. Tasa de infiltración final estable para 80 parcelas de lluvia simulada bajo lluvias de $55 \mathrm{~mm} \mathrm{~h}^{-1}$ durante 1 hora en suelos situados en margas del Cretácico (Petrer y Monnegre) y arcillas del Keuper (Toris y Requena). Se utilizó el simulador de lluvia de Cerdà et al. (1997) para cuantificar la escorrentía. La infiltración instantánea se obtuvo de la substracción de la precitación instantánea el caudal (escorrentía) instantánea.

Estas muestras fueron tomadas el verano de 1991

\begin{tabular}{|c|c|r|r|l|}
\hline Zonas & Monnegre & Petrer & Anna & Toris \\
\hline $\mathbf{1}$ & 10,21 & 18,97 & 55,00 & 44,40 \\
$\mathbf{2}$ & 18,24 & 4,25 & 41,13 & 55,00 \\
$\mathbf{3}$ & 32,58 & 12,25 & 32,91 & 54,73 \\
$\mathbf{4}$ & 27,06 & 21,75 & 55,00 & 27,75 \\
$\mathbf{5}$ & 14,85 & 20,94 & 40,54 & 50,15 \\
$\mathbf{6}$ & 28,35 & 18,58 & 45,74 & 26,35 \\
$\mathbf{7}$ & 44,91 & 23,61 & 52,71 & 55,00 \\
$\mathbf{8}$ & 12,54 & 18,85 & 28,36 & 55,00 \\
$\mathbf{9}$ & 27,82 & 19,39 & 33,82 & 38,89 \\
$\mathbf{1 0}$ & 14,25 & 17,83 & 55,00 & 35,36 \\
$\mathbf{1 1}$ & 2,32 & 27,92 & 37,28 & 29,05 \\
$\mathbf{1 2}$ & 4,25 & 25,90 & 36,38 & 55,00 \\
$\mathbf{1 3}$ & 24,35 & 20,71 & 55,00 & 43,92 \\
$\mathbf{1 4}$ & 19,58 & 20,55 & 36,79 & 47,25 \\
$\mathbf{1 5}$ & 36,93 & 20,40 & 47,76 & 37,58 \\
$\mathbf{1 6}$ & 25,59 & 5,69 & 43,15 & 37,98 \\
$\mathbf{1 7}$ & 6,06 & 23,32 & 52,79 & 27,33 \\
$\mathbf{1 8}$ & 11,82 & 5,35 & 48,98 & 47,45 \\
$\mathbf{1 9}$ & 3,65 & 24,56 & 50,20 & 50,24 \\
$\mathbf{2 0}$ & 6,35 & 5,36 & 48,88 & 55,00 \\
\hline Average & $\mathbf{1 8 , 5 8}$ & $\mathbf{1 7 , 8 1}$ & $\mathbf{4 4 , 8 7}$ & $\mathbf{4 3 , 6 7}$ \\
\hline Std & $\mathbf{1 2 , 0 2}$ & $\mathbf{7 , 2 6}$ & $\mathbf{1 0 , 4 4}$ \\
\hline & & & & \\
\hline
\end{tabular}

\section{Laderas versus pediments ¿La clave del funcionamiento geomorfológico de los badlands?}

Desde los primeros estudios geomorfológicos sobre badlands se prestó mayor atención a las laderas por su variedad de formas y procesos, por ocupar más superficie y por su espectacularidad (Sdao et al., 1984; Benito et al., 1991; Harvey y Calvo, 1991; Gar- 
cía Fayos y Cerdà, 1997). Los pediments fueron dejados de lado al ser homogéneos, no presentar formas tan llamativas y ocupar solo la parte baja al pie de las laderas, lo que habitualmente no es más del $5 \%$ del sistema badland (Fig. 7). Sin duda, el sugerente panorama de las laderas desnudas de vegetación, surcadas por regueros, con colores y formas variadas, y con evidentes signos de actividad geomorfológica explica esta preferencia. Durante décadas, y aún hoy, badlands es sinónimo de laderas desnudas, pero no de pediments. Sin embargo, al pie de las laderas se desarrollan unas superficies planas, con muy escasa cubierta vegetal, por la que transitan los sedimentos arrancados en las laderas: los pediments (Fig. 7b). Ellos son la clave de la intensa dinámica geomorfológica de las laderas de los badlands. Las altas tasas de erosión medidas en las laderas se mantienen en el tiempo porque no se produce una estabilización del pie de ladera. Es decir, no se forma coluvio que permita ralentizar el proceso de erosión en los pies de las laderas y alcanzar en ellas una forma estable, regularizada. Las laderas de los badlands mantienen su actividad geomorfológica porque nunca alcanzan la estabilidad. Así, las laderas de los badlands son rectas o convexas, debido a la importancia de la salpicadura y la arroyada superficial, y a que todo el material que llega a la base es evacuado. Si no ocurriese esto las laderas presentarían una forma cóncava, lo que no es habitual en los badlands (Hodges, 1982).

El proceso de infiltración es la clave para entender la generación de escorrentía y la erosión del regolito. Esta afirmación es general para cualquier superficie continental, pero más para los badlands donde la escasez de la vegetación reduce a la más mínima expresión la interceptación de las plantas y hojarasca (Scoging y Thornes, 1980; Dunne et al., 1991). Determina la capacidad de infiltración, su comportamiento temporal y espacial, y conocerás el funcionamiento de las laderas y los pediments en su conjunto. Este fue nuestro reto y para ello utilizamos lluvia simulada (Fig. 7B y 8), que tan buen resultado había dado en los experimentos previos, para acelerar la toma de datos respecto a la lluvia natural. Se mantuvieron las mediciones con infiltrómetro de cilindro con el objetivo de detectar los flujos preferentes en las grietas y tubos. A ello se sumó el seguimiento de la morfología de las laderas que aporto información relevante (Payà y Cerdà, 1992). La teoría de que los pediments son decisivos en el funcionamiento de los badlands requería conocer la capacidad de infiltración de los pediments y las laderas para conocer sus diferencias. El primer análisis de los resultados demostró que los pediments tienen una mayor capacidad de infiltración final estable o conductividad hidráulica (Tabla 3). Las mediciones se realizaron en los badlands de Petrer (Alicante), en la zona donde la capacidad de infiltración era menor, y donde las grietas de los badlands eran menos abundantes. Por lo tanto se evitaron los badlands con elevado contenido en montmorillonita que siempre mostraron una tendencia a generar tubos y mantener las grietas abiertas durante más tiempo, favoreciendo la infiltración concentrada en macroporos. No obstante, los experimentos mostraron como 2 de las 12 parcelas presentaban tasas de infiltración elevadas debido a la conexión de las grietas con un tubo interno durante las mediciones con infiltrómetro de cilindro. De hecho, en esos casos, y después de 30 minutos de lluvia seguían varias grietas abiertas (Fig. 8B). 
Tabla 3. Tasas de infiltración final estable medida en laderas y pediments mediante lluvia simulada ( $f c$ ) e infiltrómetro de cilindro (ifc). Muestras tomadas en el verano de 1992

\begin{tabular}{|c|c|c|c|c|}
\hline & \multicolumn{2}{|c|}{ Ladera } & \multicolumn{2}{c|}{ Pediment } \\
\hline & $f \boldsymbol{c}$ & $\begin{array}{c}\boldsymbol{i f c} \\
\left(\mathrm{mm} \mathrm{h}^{-1}\right)\end{array}$ & $\begin{array}{c}f \boldsymbol{c} \\
\left(\mathrm{mm} \mathrm{h}^{-1}\right)\end{array}$ & $\begin{array}{c}\boldsymbol{i} \boldsymbol{c} \boldsymbol{c} \\
\left(\mathrm{mm} \mathrm{h}^{-1}\right)\end{array}$ \\
\hline $\mathbf{1}$ & 6,52 & 11,25 & 25,32 & 56,32 \\
$\mathbf{3}$ & 3,25 & 12,65 & 29,32 & 67,14 \\
$\mathbf{4}$ & 4,58 & 8,35 & 19,57 & 42,36 \\
$\mathbf{5}$ & 2,45 & 9,35 & 15,25 & 38,25 \\
$\mathbf{6}$ & 6,32 & 65,32 & 12,25 & 32,57 \\
$\mathbf{7}$ & 5,36 & 11,64 & 25,32 & 67,35 \\
$\mathbf{8}$ & 4,98 & 55,32 & 14,25 & 45,68 \\
$\mathbf{9}$ & 5,65 & 16,35 & 16,32 & 56,24 \\
$\mathbf{1 0}$ & 7,25 & 14,29 & 14,02 & 42,67 \\
$\mathbf{1 1}$ & 4,25 & 12,35 & 15,24 & 45,65 \\
$\mathbf{1 2}$ & 5,36 & 12,35 & 12,36 & 35,25 \\
\hline Media & 5,25 & 9,65 & 11,25 & 36,33 \\
\hline Std & 5,10 & 19,91 & 17,54 & 47,15 \\
\hline CV (\%) & 26,46 & 19,12 & 5,98 & 11,96 \\
\hline
\end{tabular}

Con estos experimentos se confirmó la sobrevaloración de las mediciones con infiltómetro de cilindro, y se mostraron las diferencias claras entre los pediments y las laderas. Estas últimas presentan una menor capacidad de infiltración final estable debido a que una vez cerradas las grietas el flujo matricial en el suelo es prácticamente nulo $\left(5,1 \mathrm{~mm} \mathrm{~h}^{-1}\right)$. Por el contrario, el pediment mostraba aún, y después de 30 minutos de lluvia a $55 \mathrm{~mm} \mathrm{~h}^{-1}$ tasas de infiltración final estable (Ks) de $17,54 \mathrm{~mm} \mathrm{~h}^{-1}$. Esto es debido a que la falta de pendiente provocaba encharcamientos, lo que sumado a una textura limo-arenosa (Fig. 9) permitía la infiltración de las aguas (Cerdà y García Fayos, 1997). Además en los pediments suele estar presente una escasa cubierta vegetal (2-3\%) que ejerce un papel decisivo en la ralentización de los flujos y el aumento de la capacidad de infiltración. En estas condiciones, se puede prever un aumento de la sedimentación al infiltrarse las aguas procedentes de las laderas en los pediments, perder capacidad de carga por la reducción del caudal y la pendiente, y por lo tanto formar una acumulación de sedimentos al pie de la ladera: el coluvio. Sin embargo, este coluvio no existe en los pediments, no está presente en ninguna parte del sistema badland. Cuando no hay pediments a los pies de las laderas es porque hay una cárcava que drena todos los materiales, y que impide cualquier acumulación (ver Fig. 7A). 


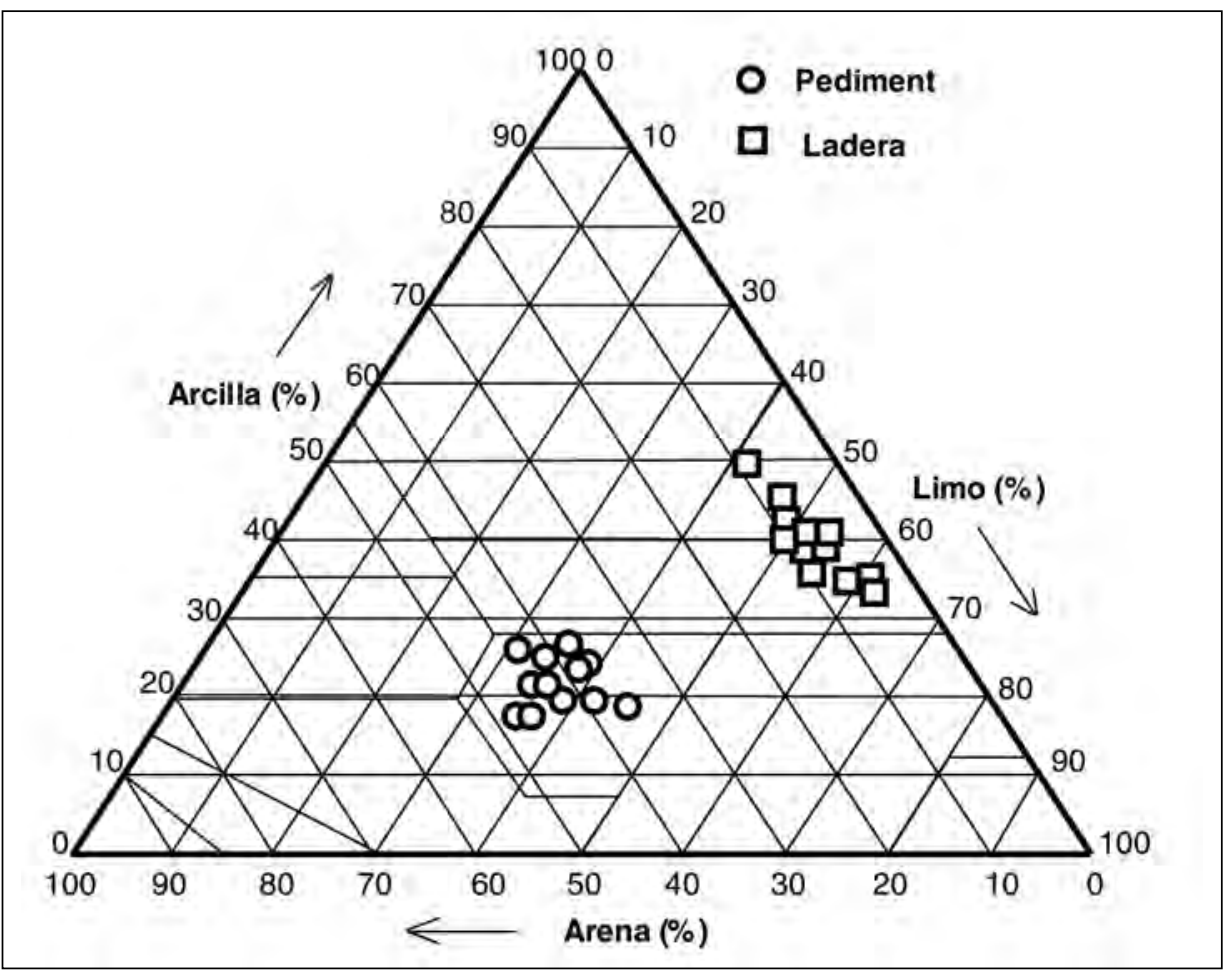

Figura 9. Textura de los pediements y laderas estudiados en la zona de Petrer

Un análisis más detallado de los hidrogramas demostró que las escorrentías se generan antes en los pediments que en las laderas (Fig. 10 y 11). Es decir, la capacidad de infiltración instantánea es inferior en los pediments que en las laderas desde el inicio del evento de lluvia, y sólo es inferior en las laderas superado el minuto 15 de la lluvia, cuando las grietas se han cerrado en su mayoría. Las mediciones tomadas durante el experimento con lluvia simulada demostraban que el encharcamiento se producía con antelación en los pediments (50,25 segundos después del inicio de la lluvia) mientras que la ladera necesitaba 82 segundos (Tabla 4). Eso se traducía en que la escorrentía en los pediments se iniciaba también antes (113 segundos frente a 129 segundos en la ladera). A pesar de que la pendiente permitía que la transformación del encharcamiento en escorrentía fuese más rápido en la ladera (32 segundos) que en los pediments (76 segundos), el inicio de la escorrentía 15 segundos antes en los pediments permite que todos los materiales llegados desde la ladera sean rápidamente evacuados al encontrar superficies como los pediments: saturados y en los que el transporte de materiales es muy eficiente. A ello también ayuda el que la concentración de sedimentos en los pediments sea muy baja ( $\left.2 \mathrm{gr} \mathrm{l}^{-1}\right)$ mientras que en las laderas supera los 10 e incluso los $50 \mathrm{~g} \mathrm{l}^{-1}$, de ahí que el material disperso (agregados dispersados al contacto con el agua debido al alto contenido en sodio, ver Shainberg et al., 1981; Imeson y Verstraten, 1988; Gerits, 1991; 


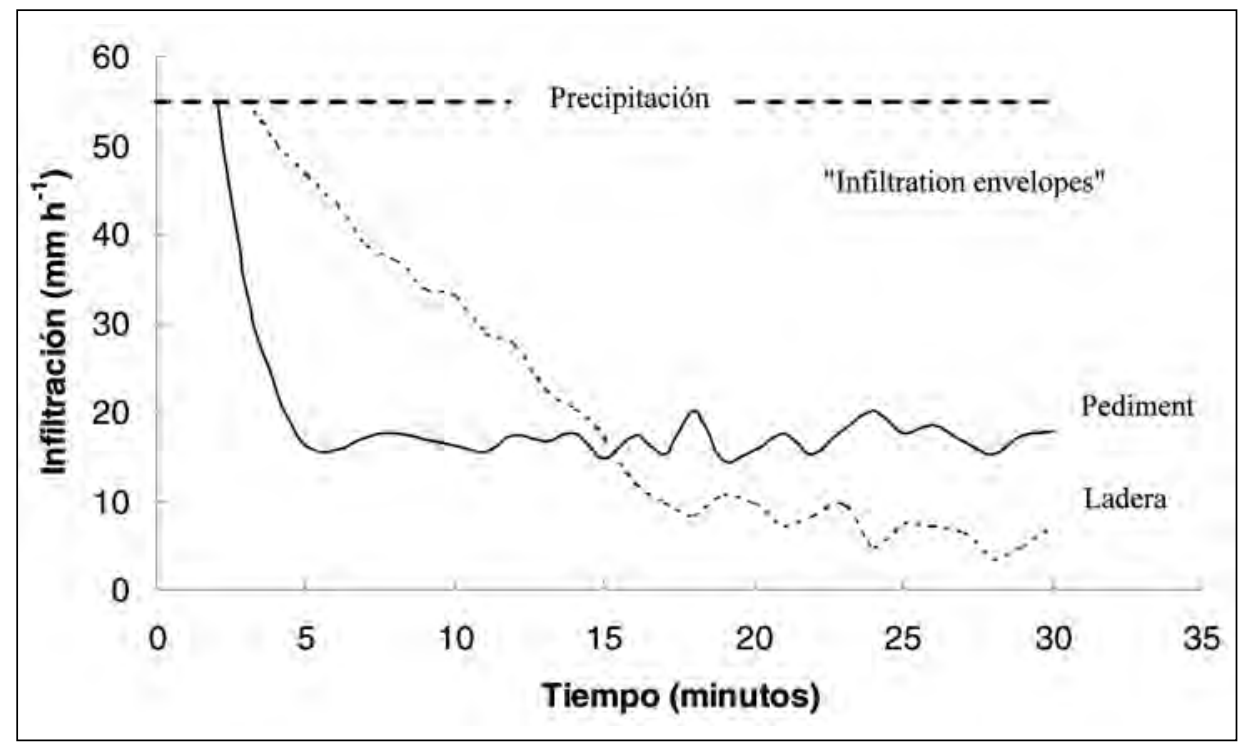

Figura 10. Curvas de infiltración medias para los 12 experimentos en pediments y los 12 en laderas ejecutados en los badlands de Petrer. Se puede apreciar la rápida respuesta de obtenida en los pediments, aunque muestren al final una capacidad de infiltración final estable superior a la de las laderas

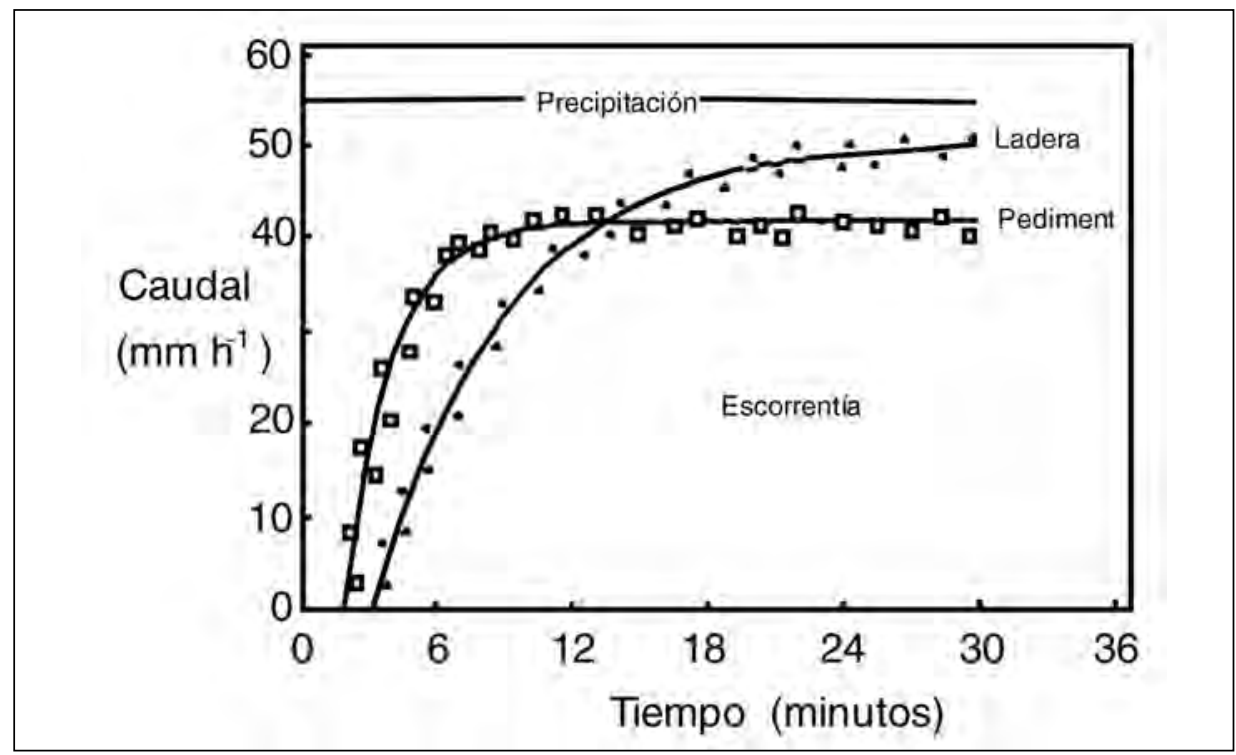

Figura 11. Hidrogramas de la escorrentía en dos parcelas-pediment y ladera-en los badlands de Petrer (Alicante). Se muestra el rápido inicio de la escorrentía y su estabilización en los pediments, proceso que es más lento y retrasado en las laderas debido a la presencia de grietas 
Tabla 4. Tiempo de encharcamiento (tp), tiempo de escorrentía (tr) y tiempo de retraso (tp-tr) para 12 parcelas en las laderas y 12 en los pediments de los badlands del Petrer.

Muestras tomadas en el verano de 1992

\begin{tabular}{|c|c|c|c|c|c|c|}
\hline \multirow[t]{2}{*}{ Parcelas } & \multicolumn{3}{|c|}{ Ladera } & \multicolumn{3}{|c|}{ Pediment } \\
\hline & $\begin{array}{c}t p \\
\text { segundos }\end{array}$ & $\begin{array}{c}\boldsymbol{t r} \\
\text { segundos }\end{array}$ & $\begin{array}{c}\boldsymbol{t p} \text {-tr } \\
\text { segundos }\end{array}$ & $\begin{array}{c}\boldsymbol{t p} \\
\text { segundos }\end{array}$ & $\begin{array}{c}\boldsymbol{t r} \\
\text { segundos }\end{array}$ & $\begin{array}{c}t p-t r \\
\text { segundos }\end{array}$ \\
\hline 1 & 75 & 200 & 125 & 50 & 180 & 130 \\
\hline 2 & 78 & 145 & 67 & 55 & 128 & 73 \\
\hline 3 & 85 & 133 & 48 & 54 & 112 & 58 \\
\hline 4 & 60 & 100 & 40 & 45 & 90 & 45 \\
\hline 5 & 85 & 102 & 17 & 40 & 96 & 56 \\
\hline 6 & 75 & 135 & 60 & 55 & 118 & 63 \\
\hline 7 & 74 & 142 & 68 & 45 & 129 & 84 \\
\hline 8 & 79 & 98 & 19 & 58 & 68 & 10 \\
\hline 9 & 100 & 132 & 32 & 60 & 110 & 50 \\
\hline 10 & 99 & 112 & 13 & 49 & 113 & 64 \\
\hline 11 & 86 & 135 & 49 & 49 & 125 & 76 \\
\hline 12 & 92 & 124 & 32 & 43 & 95 & 52 \\
\hline Media & 82,33 & 129,83 & 47,5 & 50,25 & 113,67 & 63,42 \\
\hline Std & 11,35 & 27,63 & 30,80 & 6,25 & 27,49 & 28,11 \\
\hline CV $(\%)$ & 13,78 & 21,28 & 64,84 & 12,45 & 24,18 & 44,33 \\
\hline
\end{tabular}

Benito et al., 1993) en las laderas no se sedimente en los pediments sino que fluye fuera del sistema y alcanza los cauces. Las curvas de infiltración (Fig. 10) y las curvas de escorrentías (Fig. 11) demuestran este comportamiento de los pediments y badlands que será clave para el proceso de erosión.

Los resultados demuestran que los pediments son superficies de transporte en los que hay puntualmente sedimentación o erosión, mientras que en las laderas de los badlands la pérdida de material es continua. Los pediments presentan una capacidad de infiltración extraordinariamente baja desde el principio de la lluvia, incluso inferior a la de las laderas de badlands mientras se mantienen en estos las grietas abiertas. Pasados 15 minutos esta situación se invierte. Por ello, lluvias intensas y cortas hacen que los pediments sean altamente eficientes en el transporte de material. Además, mientras los pediments son muy homogéneos en su respuesta hidrológica, las laderas son extraordinariamente heterogéneas. En los pediments, al presentar escasas y superficiales grietas la generación de escorrentía es inmediata, al contrario que en las laderas que el cierre de 
grietas hace que la escorrentía se genere de forma progresiva (Fig. 12). La tabla 4 muestra la rapidez con que se produce la generación de escorrentías en los pediments y la Fig. 10 presenta la forma típica de las curvas de infiltración en las que se comprueba como el pediment alcanza rápidamente la estabilidad y la máxima capacidad de infiltración (5 minutos) mientras que las laderas necesitan 20 minutos para alcanzar los valores máximos, fruto de la infiltración en las grietras mientras estén abiertas.

El estudio de los pediments y laderas demostró que los badlands lejos de ser superficies homogéneas presentan una elevada variabilidad espacial en la generación de sedimentos y escorrentías lo que está controlado por la presencia de los pediments en los pies de las laderas. Son menos del $5 \%$ de las superficies de los badlands, pero de su reducida capacidad de infiltración desde el inicio de la lluvia como consecuencias de la escasez de grietas depende su eficiencia en el trasnporte de materiales. Estos resultados apuntan a que la clave en la estabilización de los badlands está en la acumulación del material erosionado al pie de la ladera, donde se encuentra el pediment. El reto en la investigación de los próximos años está en desarrollar estrategias que permitan la estabilización de los pediments cuando sea ambientalmente necesario. Tal vez la vegetación sea la clave para ello (García Fayos et al., 1995; Regüés et al., 2000).

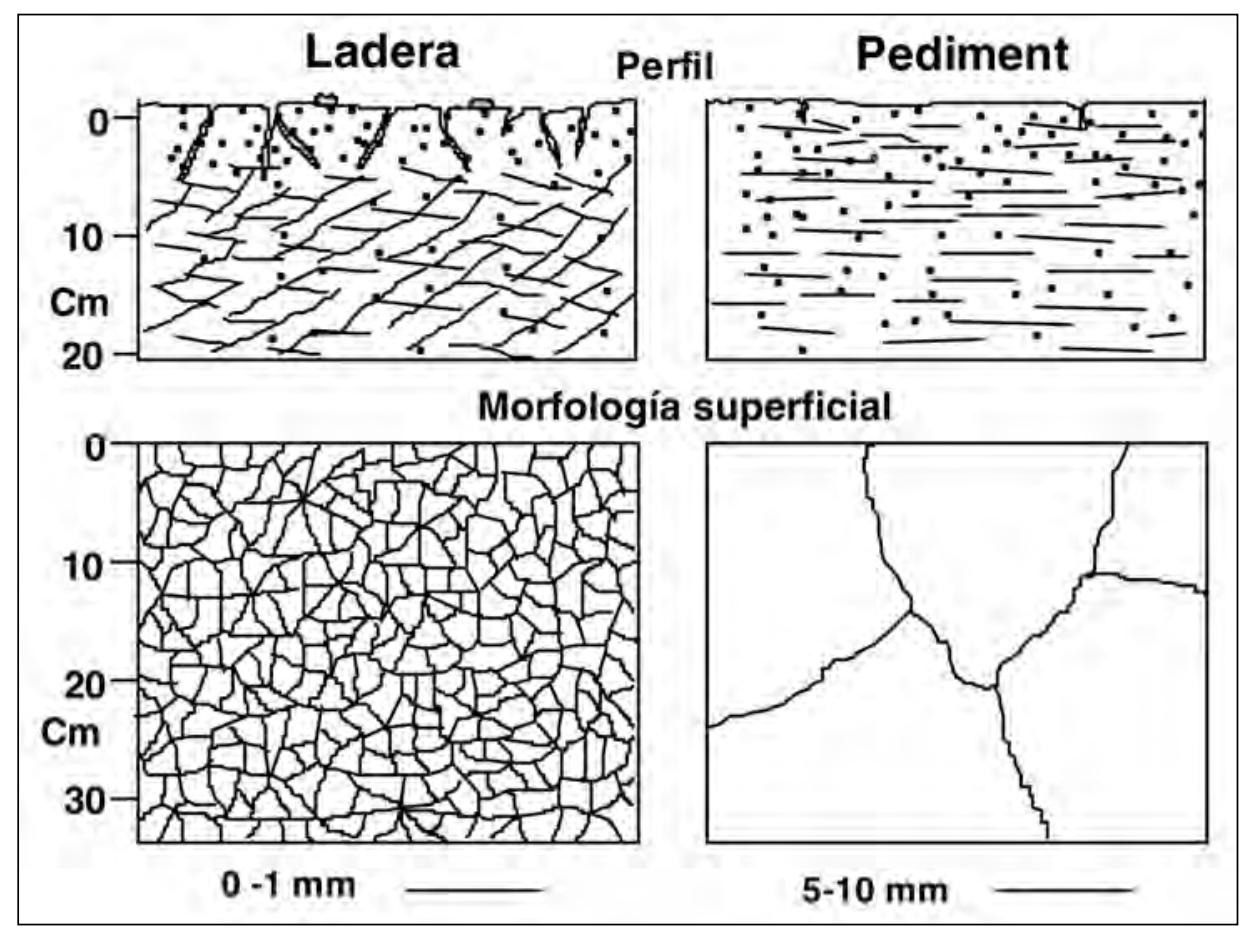

Figura 12. Perfil y morfología superficial de los pediments y laderas de badlands. La presencia de grietas abundantes, profundas y amplias condiciona la infiltración y la generación de la escorrentías 
Los retos de la investigación para el futuro son: (i) estudiar con más detenimiento la conexión de las grietas y los macroporos internos, habitualmente situados entre la roca madre y el regolito superficial, para conocer la partición de la lluvia en escorrentía superficial y subsuperficial. Es decisivo en este aspecto desarrollar un modelo dinámico dependiente de la precipitación, ya que obedeciendo a la duración del evento tendremos lluvia infiltrada (primeros cinco minutos), escorrentía superficial drenando desde las zonas de entre-grietas a las grietas donde se comunica con las tubificaciones del minuto 5 al 15, y finalmente una impermeabilización de la superficie pasados 15 minutos. Aquellos escasos pero existentes macroporos abiertos después de 15 minutos pueden absorber un caudal elevado drenado desde las superficies saturadas de los badlands, y aquí puede estar el origen de las tubificaciones. (ii) Esta aproximación temporal al proceso de infiltración debe ir acompañado de un estudio espacial (a distintas escalas) bajo lluvia natural. Esta aproximación ya ha sido parcialmente investigadas por la Estación Experimental de Zonas Áridas de Almería (Cantón et al., 2001) pero a escala de cuenca de drenaje. Ese mismo estudio a escala de ladera, desde mediciones entre grietas (10 $\mathrm{cm})$, entre-regueros $(100 \mathrm{~cm})$ o entre cárcavas $(1000 \mathrm{~cm})$ podría aportar una respuesta definitiva a las preguntas que hoy no podemos resolver: a) como se conecta la lluvia y la escorrentía infiltrada con la red de tubificaciones internas de los badlands; y b) si hay un retorno de flujo a la superficie como en ocasiones hemos visto en experimentos con lluvia simulada. El desarrollo de los modelos debe estar basado en un amplio y prolongado seguimiento de parcelas bajo lluvia natural para confirmar lo apuntado aquí mediante la lluvia simulada.

\section{La estacionalidad de la infiltración en los badlands}

Otra idea preconcebida sobre la hidrología de los badlands es que presentan escasas variaciones temporales y estacionales. Lejos de ser superficies homogéneas y sin cambios en el tiempo, los badlands se muestran con una dinámica hidrológica extraordinariamente activa. La estacionalidad morfológica, y sus implicaciones hidrológicas en los badlands ha sido ampliamente estudiada por David Regüés en el Pirineo Catalán (Regüés et al., 1995; Regüés y Gallart, 2004) y aragonés (Nadal Romero et al., 2006) donde se presentan distintos factores determinantes de una contrastada respuesta hidrológica y erosiva: helada, humectación-desecación, tiempo desde la última lluvia, etc. En los badlands del sudeste peninsular donde las heladas son escasas y los procesos de humectación-desecación reducidos al producirse menos de 40 días de lluvias y volúmenes inferiores a $350 \mathrm{~mm}$ año-1 de precipitación, la estacionalidad de la producción de sedimentos y caudales está básicamente controlada por el regolito disponible y la morfología de la superficie donde las grietas juegan un papel preponderante (Cerdà, 1996b). Es por lo tanto, el tiempo transcurrido desde la última lluvia un factor esencial en la carga sedimentaria y también en la capacidad de infiltración de los suelos. Obviamente, el roquedo también es decisivo en este aspecto, ya apuntado en el apartado anterior (Cerdà, 1999c), como también lo es la exposición (Churchill, 1981; Cerdà, 1996a), aunque aquí no es tratado. 
Las mediciones y experimentos desarrollados en las estaciones experimentales de Petrer, Toris, Monnegre, Anna y Requena, permitió confirmar un ciclo anual de la capacidad de infiltración, que a su vez determinaba la producción de sedimentos y escorrentías (Cerdà, 1999a). Durante los periodos húmedos desde Octubre hasta Junio, los suelos de los badlands presentan niveles de humedad variables dependiendo de la recurrencia de las precipitaciones (Cerdà, 1996b). Por el contrario, la sequía estival mediterránea transforma el regolito en un substrato sin agua $(<2 \%)$ lo que hace imposible su regeneración al concentrarse las sales e impedir la viabilidad de las plántulas (García Fayos et al., 2000).

La zona de estudio de Petrer se encuentra en el oeste de la provincia de Alicante (Fig. 13), con precipitaciones medias anuales inferiores a $300 \mathrm{~mm}$, y alta evapotranspiración (Fig. 14-16). En ella la estacionalidad climática es elevada al alcanzar la sequia estival los 5 meses. La evolución de la infiltración se muestra de acuerdo con estas condiciones, siendo mayor en verano que en el resto de estaciones. La presencia de grietas es decisiva como hemos en la hidrología de los badlands y estas a su vez dependen de la humedad. El irregular clima mediterráneo puede provocar condiciones áridas en pleno invierno, pero la década de estudio en las cinco zonas antes mencionadas siempre mostraban claras diferencias entre el verano y el resto del año en cuanto a tamaño y número de grietas, capacidad de infiltración, material disponible de ser erosionado, tiempo de encharcamiento o tiempo de inicio de la escorrentía.

Las mediciones realizadas en los badlands de Petrer sobre margas durante el año 1990 demostraron que los suelos se encharcaban (se alcanzaba la capacidad de infiltración máxima) rápidamente en otoño, invierno y primavera, y que esto ocurría siempre de forma más retrasada en verano. En esta estación el encharcamiento en los badlands se alcanzaba después de 174 segundos y la escorrentía tras 250 segundos. Para los pediments fue de 102 y 140 segundos respectivamente. Estas mismas superficies durante el resto del año contribuían con encharcamientos tras 80-110 segundos y escorrentías tras 113-138 segundos. Para los pediments fueron 34-49 y 55-73 segundos respectivamente (Fig. 17 y 18). Por lo tanto, las escorrentías se producen mucho más lentamente en verano que en el resto del año lo que es fruto de tasas de infiltración más altas, no sólo en los momentos iniciales de la lluvia, sino que incluso afectaron claramente a la capacidad de infiltración final estable al mantenerse grietas abiertas en algunas superficies.

Así, los valores de la tasas de infiltración final estable, tanto para pediments como para laderas, está controlada por el contenido de humedad del suelo que a su vez determina la presencia de grietas y de su tamaño. En ambos casos, pediments y laderas, las tasas de infiltración se duplican en verano respecto al resto de estaciones como consecuencia de la escasa humedad del suelo. De hecho, los valores medidos muestran una duplicación de la tasa de infitración final estable o conductividad hidráulica saturada del suelo en verano. La razón de que se modifique estos parámetros $(f c$ o $K s)$ que son entendidos como estables o permanentes en el suelo, se debe a que el regolito en verano presenta unas condiciones muy distintas. En él se pueden apreciar más macroporos, no sólo la superficie del regolito (más desarrollado y profundo después de meses sin erosión por 


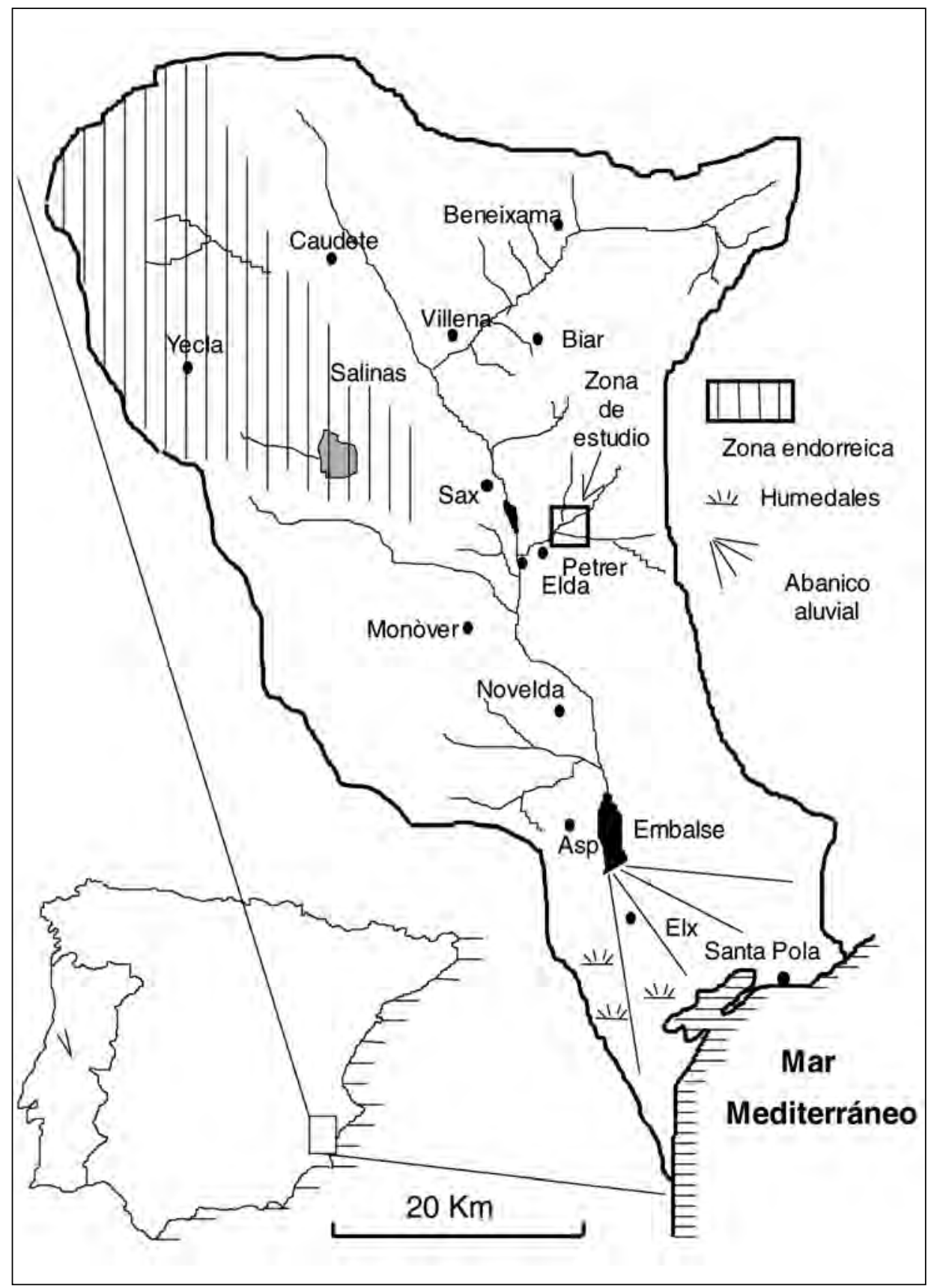

Figura 13. Localización de la zona de estudio de Petrer, Alicante 


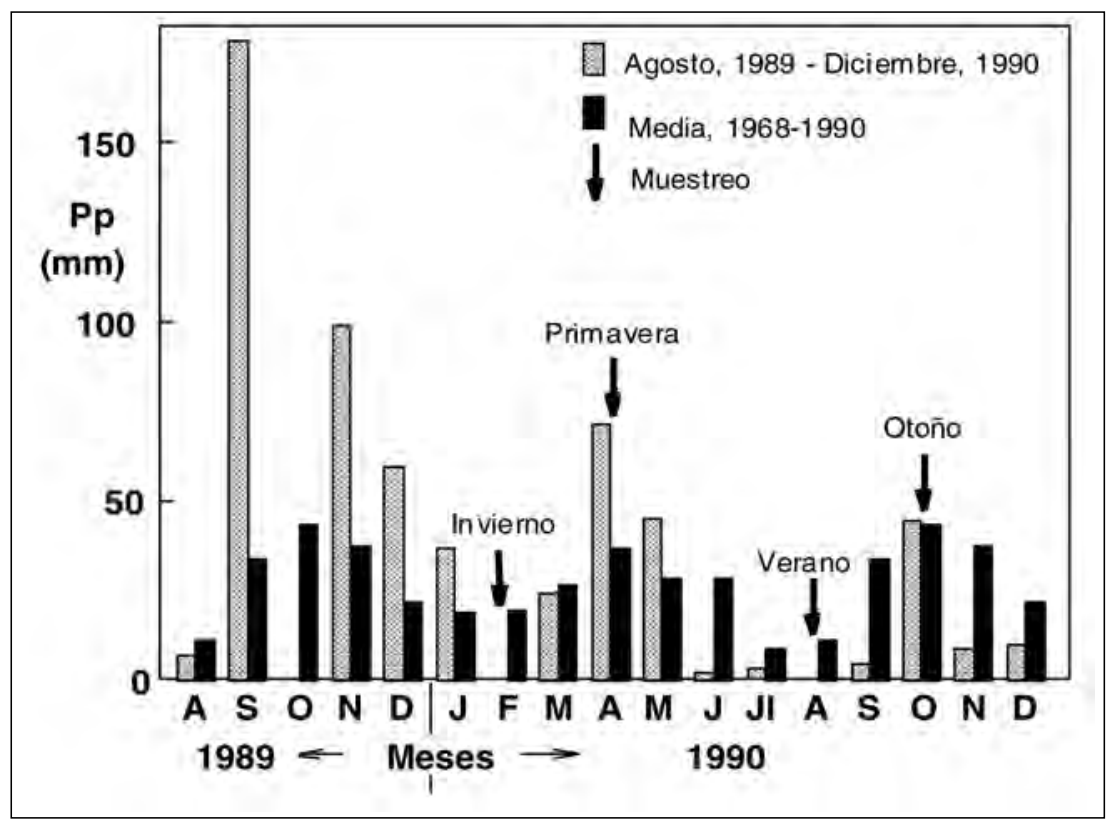

Figura 14. Precipitación mensual durante el periodo de esudio y en la serie entre 1968 y 1990 en Petrer

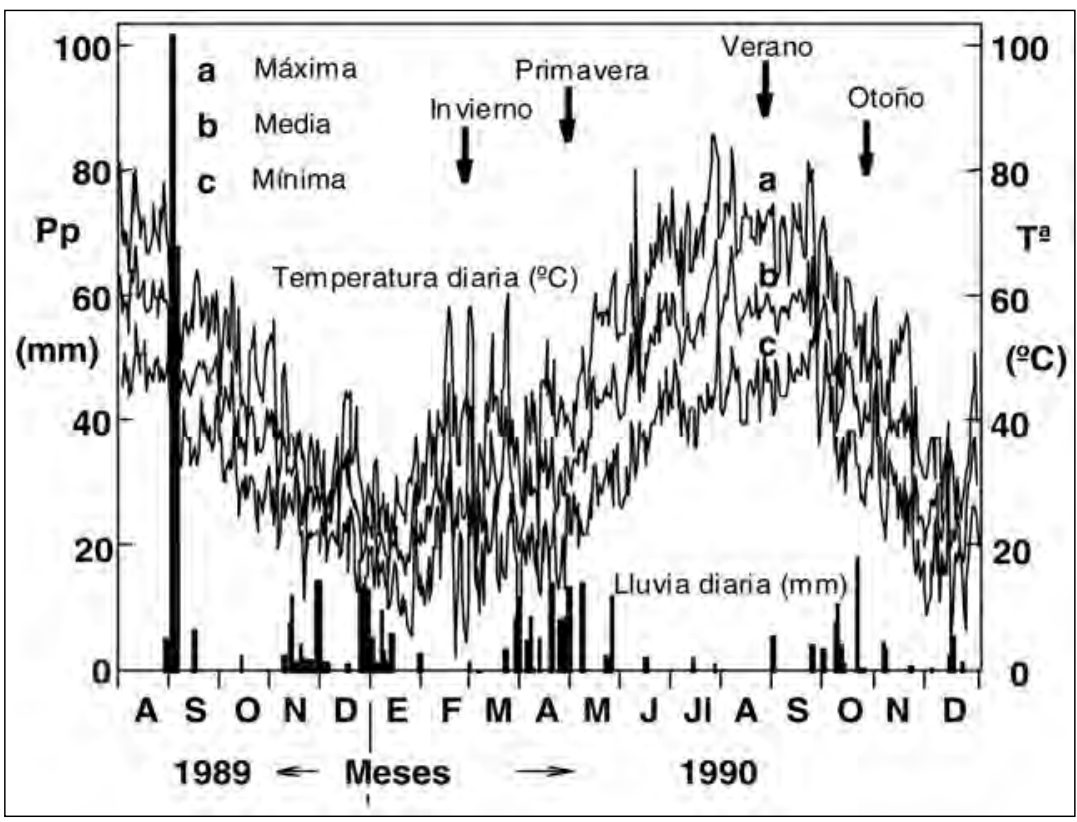

Figura 15. Precipitación y temperatura (media, maxima y mínima diaria) durante el periodo de estudio en el año 1990. Destaca la falta de heladas y las reducidas precipitaciones 

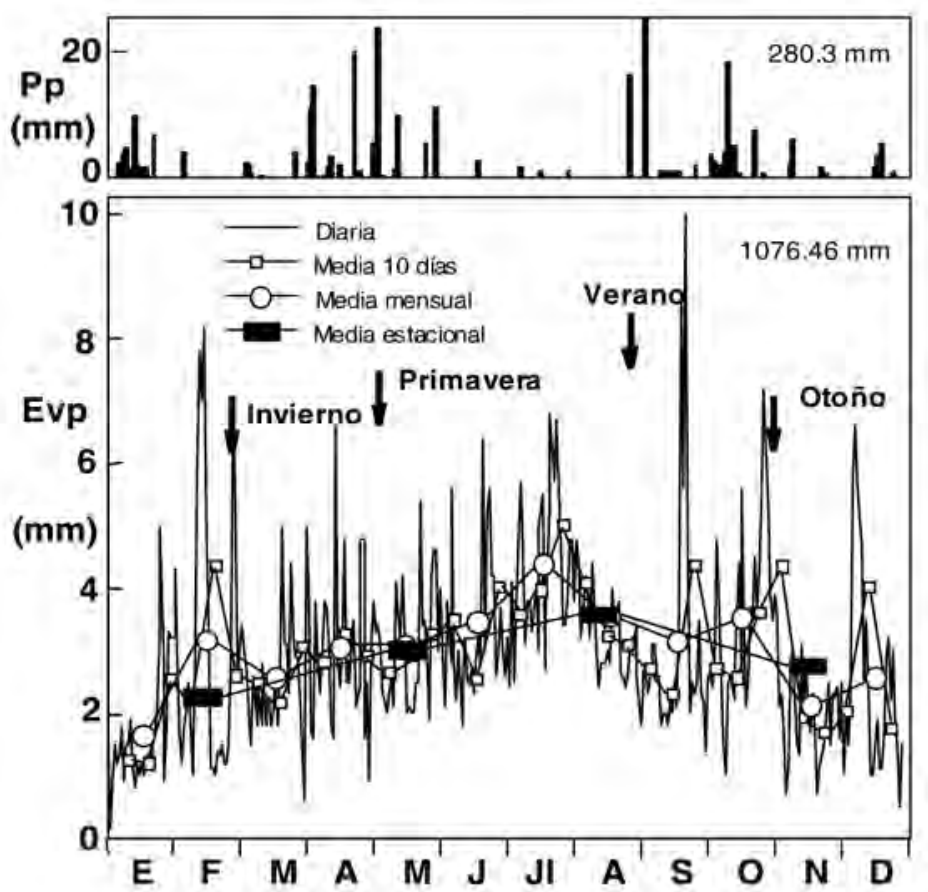

1990

Figura 16. Precipitación diaria y evaporación (diaria, media de 10 días, media mensual y estacional) para la zona de estudio de Petrer. Es de destacar que la evaporación es cuatro veces mayor a la precipitación

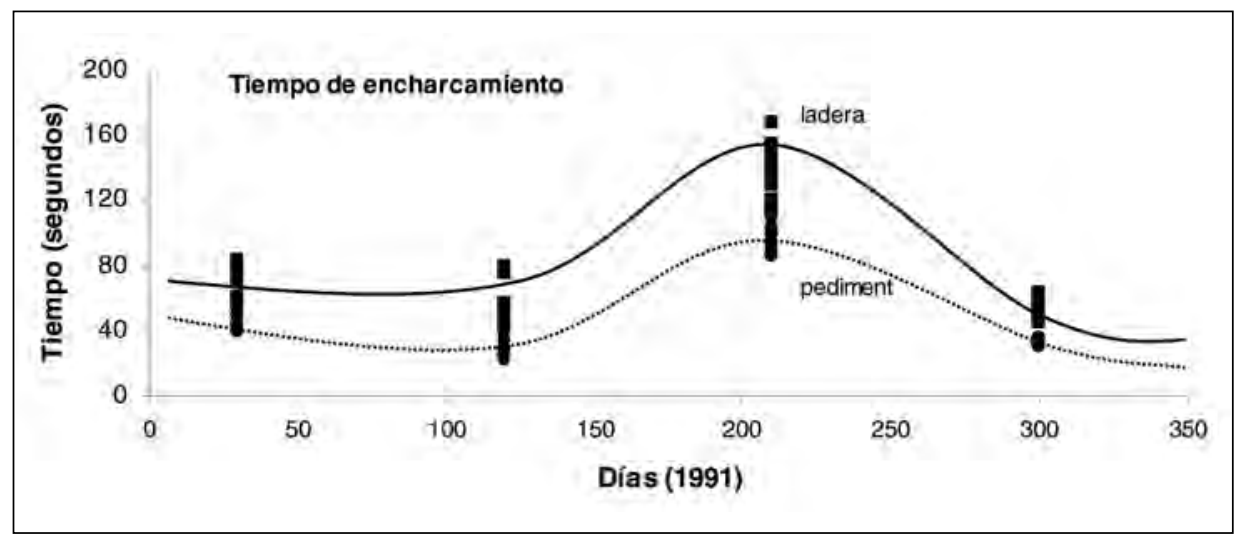

Figura 17. Tiempo de escharcamiento (tp) para 10 parcelas de pediments y 10 de laderas durante las cuatro estaciones. Datos para el año 1991 


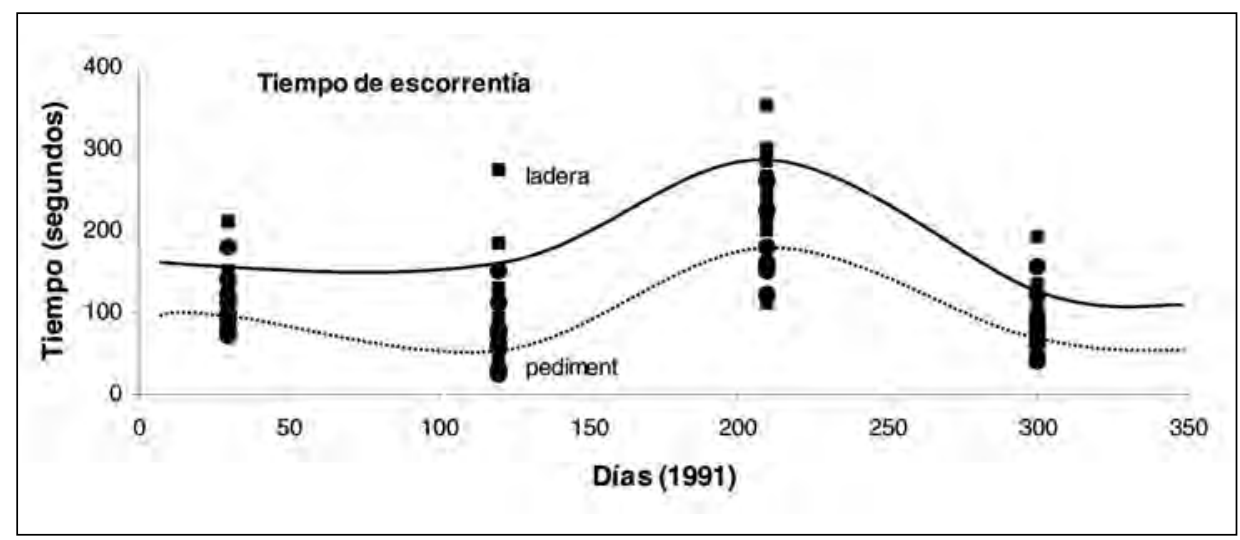

Figura 18. Tiempo de escorrentía (tr) para 10 parcelas de pediments y 10 de laderas durante las cuatro estaciones. Datos para el año 1991

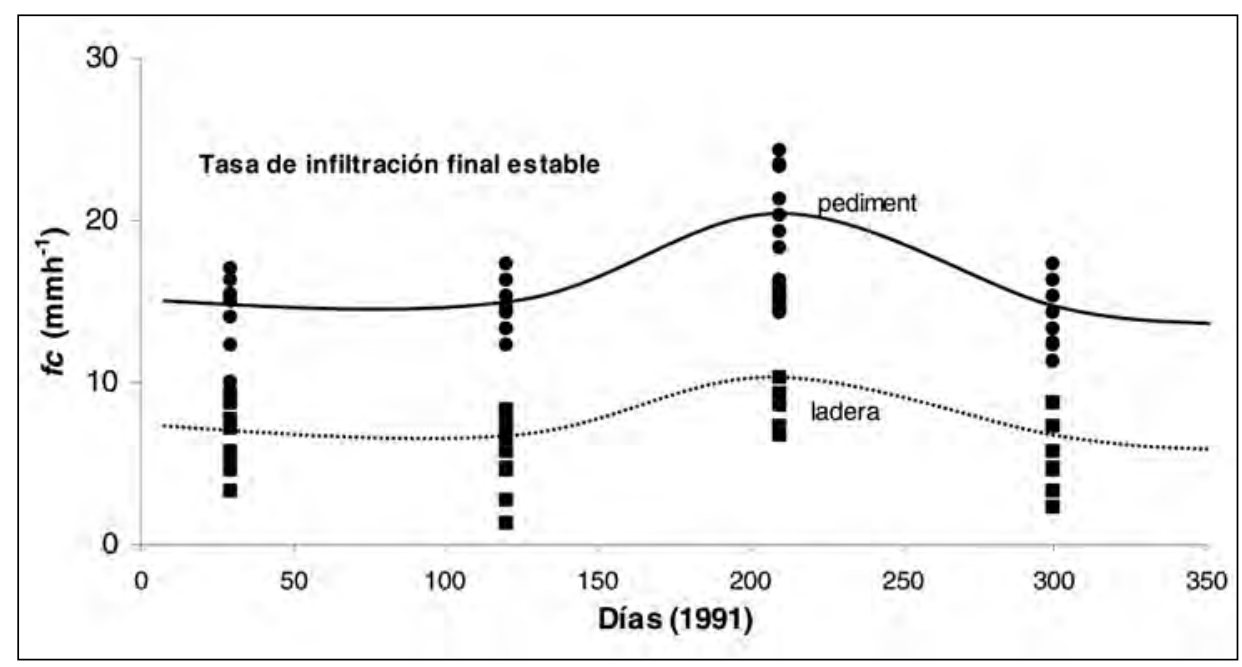

Figura 19. Evolución estacional de la tasa de infiltración final estable para pediments y badlands de la estación experimental de Petrer. Tiempo de escharcamiento (tp) y tiempo de escorrentía (tr) para 10 parcelas de pediments y 10 de laderas durante las cuatro estaciones.

Datos para el año 1991

falta de lluvia) presenta grietas profundas, sino que estas se conectan con zonas de debilidad (fracturas fruto de la descompresión o de movimientos internos), propias de la roca madre, y esto permite que la infiltración sea más profunda, y la responsable de la formación de nuevos tubos que drenan en profundidad parte de la precipitación y también de la escorrentía generada entre grietas. Esto permite que en algunas zonas la escorrentía finalmente no corra en superficie sino que drene hacia las grietas internas. 
Estos resultados confirman que la formación de los tubos (piping) en los badlands debe de originarse como consecuencia de líneas de debilidad de la roca madre o en su contacto con el regolito. Esas líneas de debilidad son acentuadas con los procesos de hinchamiento-deshinchamiento del material, pero que será la infiltración de las aguas desde la superficie en las laderas las que conformarán la amplia red de drenaje subsuperficial que es a su vez la que consigue formar crecidas con caudales pico extraordinarios. En ello tiene mucho que decir la velocidad del flujo a presión en macroporos, otro tema relevante que deberá ser estudiado en el futuro, como todo lo relacionado con el piping, del cual conocemos bien poco.

\section{Conclusiones}

La infiltración en los badlands es muy variable espacial, temporal y estacionalmente debido a la morfología superficial. Son las grietas y las conexiones de estas con el flujo subsuperficial las que determinan la hidrología de los badlands. Es de destacar que el verano es siempre la estación en la que la capacidad de infiltración es mayor ya que también es cuando las grietas son mayores, fruto de la escasa humedad. Destaca la reducida capacidad de infiltración de los pediments desde el principio de la lluvia lo que los convierte en superficies extraordinariamente eficientes en el vehiculación de materiales procedentes de las laderas. Y que los pediments y las laderas tienen comportamientos hidrológicos distintos permite la elevada actividad erosiva de los badlands a los pocos minutos de iniciada la lluvia. Los métodos empleados -infiltrómetro de cilindro y lluvia simulada- han sido complementarios y ambos han permitido conocer mejor la hidrología de los badlands. Sin embargo, aconsejamos la lluvia simulada para el estudio de estas superficies en las que el impacto de las gotas, la salpicadura, las costras y las grietas son decisivos en el proceso de infiltración.

Esta revisión del proceso de infiltración quiere incidir en los puntos débiles de nuestros conocimientos: (i) conexión entre la escorrentía superficial y subsuperficial; (ii) efecto de la escala en el proceso de generación de escorrentía y producción de sedimentos en las laderas de los badlands y su conexión con los pediments; y (iii) monitorización de los procesos hidrológicos y erosivos para la búsqueda de soluciones al control de la erosión en otros espacios con un dinámica hidrogeomorfológica similar como son los taludes de carretera, derrubios de minas, desmontes, suelos agrícolas o suelos afectados por incendios. Los badlands deben quedar como laboratorios geomorfológicos y paisajes protegido.

\section{Agradecimientos}

El estudio de los badlands desde una perspectiva hidrológica siempre fue un tema secundario en la literatura geomorfológica, especialmente en la española. Durante décadas el proceso de infiltración fue obviado, en parte por la dificultad de medirlo. El profesor Adolfo Calvo fue quien sembró el interés por este aspecto en el que la colabora- 
ción con otros investigadores (Adrian Harvey, Roy Alexander o Jorge Payà) permitió al primero de los autores desarrollar la investigación aquí presentada durante los años 90. Los trabajos en el campo no se hubieran podido ejecutar sin la ayuda de varios colaboradores, a los cuales debemos nuestra gratitud por las duras jornadas de trabajo y el buen humor que siempre estuvo presente.

\section{Referencias bibliográficas}

Agassi, M., Shainberg, I., VAn Der Merwe, D. (1994). Effect of water salinity on inter-rill erosion and infiltration: laboratory study. Aust. J. Soil Res., 32: 595-601.

AlEXANDER, D. (1982). Difference between "calanchi" and "biancane" badlands in Italy. En: Badland geomorphology and piping (Bryan, R., Yair, A., Eds.). University Press, pp. 71-88, Cambridge.

Alexander, R.W., CAlvo, A. (1990). The influence of lichens on slope processes in some Spanish Badlands. En: Vegetation and Erosion: Process and Environments (Thornes, J.B., Ed.). pp. 385-98.

Alexander, R.W., Harvey, A.M., Calvo, A., James, P.A., CerdÀ, A. (1994). Natural stabilisation mechanisms on badland slopes: Tabernas, Almería, Spain. En: Effects of Environment Change in Drylands (Millington, A.C., Pye, K., Eds). pp. 85-111.

Arnáez, J., Lasanta, T., Ruiz Flaño, P., OrTigosa, L. (2007). Factors affecting runoff and erosion under simulated rainfall in Mediterranean vineyards. Soil \& Tillage Research: 93, 324-334.

BEGUERÍA, S. (2005). Identificación y características de las fuentesde sedimento en áreas de montaña: erosión y transferencia de sedimento en la cuenca alta del río Aragón. Instituto Pirenaico de Ecología, Consejo Superior deInvestigaciones Científicas, 158 pág., Zaragoza.

Benito, G., Gutiérrez, M., SANChO, C. (1991). Erosion patterns in rill and interill areas in badlands zones of the middle Ebro basin (N.E-Spain). En: Soil Erosion Studies in Spain (Sala, M., Rubio, J.L., García Ruiz, J.M., Eds.). pp. 41-54.

Benito, G., GutiÉrRez, M., SANCHO, C. (1993). The influence of physico-chemical properties on erosion processes in badland areas, Ebro basin, NE-Spain. Z. Geomorph., 37: 199-214.

BERNDTSSON, R., HOGLAND, W., LARSON, M. (1985). Spatial variations of infiltration for a catchment in northern Tunisia. En: Hydrological studies in Tunisia (Berndtsson, R., Larson, M., Dahlblom, P., Nguyen, V.L., Hogland, W., Niemczynowicz, J., Eds). pp. 13-26.

BRYAN, R., YAIR, A. (1982a). Badland geomorphology and piping (Bryan, R., Yair, A., Eds.). University Press, 409 pág., Cambridge. 
BRYAN, R., YAIR, A. (1982b). Perspectives on studies of badland geomorphology. En: Badland geomorphology and piping (Bryan, R., Yair, A., Eds.). University Press, pp. 1-12, Cambridge.

BRYAN, R.B. (1974). Water erosion by splash and wash and the erodibility of Albertan soils. Geografiska Annaler, 56 A: 159-181.

BRYAN, R.B. (1981). Soil erosion under simulated rainfall in the fields and laboratory: variability of erosion under controlled conditions. IAHS Publication, 133: 391-402.

Bryan, R.B., Imeson, A.C., CAMPBell, I.A. (1984). Solute realease and sediment entrainment on microcatchments in the Dinosaur Park Badlands Alberta, Canada. Journal of Hydrology, 71: 79-106.

Calvo, A., Harvey, A.M., PayÀ, J. (1991a). Process interactions and badland development in SE Spain. En: Soil Erosion Studies in Spain (Sala, M., Rubio J. L., García Ruiz, J. M. Eds.). Geoforma Ediciones, pp. 75-90, Logroño.

Calvo, A., Harvey, A.M., PayÀ, J., Alexander, R.W. (1991b). Response of badland surfaces in South East Spain to simulated rainfall. Rev. $C \& G, 5: 3-14$.

CAMPBELL, I.A. (1974). Erosion rates in the Steveville badlands, Alberta. Z. Geomorph. N.F. Suppl. Bd., 21: 122, 137.

CAMPBELL, I.A. (1982). Badland and Badland Gullies. En: Arid Zone geomorphology (Thomas, D.S.G., Ed.). pp. 159-186.

CAMPBELL, I.A. (1987). Infiltration Characteristics of Badlands Surface and Storm Runoff. En: Pre-Conference Proceedings ot the International Conference on Infiltration Development and Application (Fok, Y. F., Ed.). pp. 251-261.

CAMPBELL, I.A. (1989). Badlands and badland gullies. En: Arid Zone Geomorphology (Thomas, D.S.G., Ed.). pp. 159-186.

Cantón, Y., Domingo, F., Solé Benet, A., Puigdefábregas, J. (2001). Hydrological and erosion response of a badlands system in semiarid SE Spain. Journal of Hydro$\log y, 252$ : 65-84.

Cantón, Y., Del Barrio, G., Solé Benet, A., LÁZAro, R. (2004). Topographic controls on the spatial distribution of ground cover in the Tabernas badlands of SE Spain. Catena: 55, 341-365.

CERDÀ, A. (1993a). Metodologías para el estudio de la hidrología y erosión de superficies degradadas (badland). a partir de lluvia simulada. Rev. $C \& G, 7: 35-48$.

CERDÀ, A. (1993b). La infiltración en los suelos del País Valenciano. Factores y variaciones espacio-temporales. Tesis Doctoral. Facultad de Geografía e Historia, Universitat de València, 375 pág., Valencia. 
CERDÀ, A. (1995a). Hidrología y erosionabilidad de los suelos en ambientes semi-áridos. Petrer, Alicante. Studia Oecologica, XII: 159-164.

CERDÀ, A. (1995b). Factores y variaciones espacio-temporales de la infiltración en los ecosistemas mediterráneos. Monografías Científicas 5, Geoforma Ediciones, 151 pág., Logroño.

CERDÀ, A. (1996a). Régimen hídrico de los suelos en zonas acarcavadas. Algunos ejemplos de la cuenca experimental de Petrer. Alicante. Cuadernos do Laboratorio Xeolóxico de Laxe, 21: 685-694.

CERDÀ, A. (1996b). Seasonal variability of infiltration rates under contrasting slope conditions in southeast Spain. Geoderma, 69: 217-232.

CERDÀ, A. (1997). Influencia de la litología en los procesos de erosión en badlands. Los casos de Anna (Valencia) y Petrer (Alicante). Pirineos, 149-150: 3-20.

CERDÀ, A. (1999a). Seasonal and spatial variations in infiltration rates in badland surfaces under Mediterranean climatic conditions. Water Resources Research, 35: 319-328.

CERDÀ, A. (1999b). Simuladores de lluvia y su aplicación a la Geomorfología. Estado de la cuestión. Cuadernos de Investigación Geográfica, 25: 45-84.

CERDÀ, A. (1999c). Parent material and vegetation affect soil erosion in eastern Spain. Soil Science Society of America Journal, 63: 362-368.

CERDÀ, A. (2007). Soil water erosion on road enbankments in eastern Spain. Science of the Total Environment, 378: 151-155.

CERdÀ, A., García FAyOS, P. (1994/1995). Relación entre la pérdida de agua, suelo y semillas en zonas acarcavadas. Influencia de la pendiente. Cuadernos de Investigación Geográfica, 20-21: 30-45.

CERDÀ, A., PAYÀ, J. (1995). Morfología y procesos superficiales en badlands sobre arcillas del Keuper, Requena, Valencia. Rev. $C \& G$, 9: 59-72.

CERDÀ, A., GARCÍA FAYOS, P. (1997). The influence of slope angle on sediment, water and seed losses on badland landscapes. Geomorphology, 18: 77-90.

Cerdà, A., NAVArro, R. (1997). Procesos de erosión en los badlands alicantinos. Investigaciones Geográficas, 17: 99-116.

CERdÀ. A., IBÁÑEZ, S., CALVo, A. (1997). Design and operation of a small and portable rainfall simulator for rugged terrain. Soil Technology, 11: 163-170.

CERDÀ, A., DoERr, S.H. (2007). Soil wettability, runoff and erodibility of major dryMediterranean land use types on calcareous soils. Hydrological Processes, 21: 23252336. 
CHuRCHILl, R.R. (1981). Aspect-related differences in badlands slope morphology. Annals of the Association of American Geographers, 71: 374-388.

Clotet, N., Gallart, F., SAla, M. (1987). Los badlands: características, interes teórico, dinámica y tasas de erosión. Notas de Geografía Física , 1: 15-16.

DUNIN, F.X. (1976). Infiltration: its simulation for field conditions. En: Facets of Hydrology (Rodda, J.C., Ed.). John Wiley and Sons, pp. 199-227, Bristol.

Dunne, T., ZHANG, W., AUBRY, B.F. (1991). Effects of rainfall, vegetation and microtopography on infiltration and runoff. Water Resources Research, 27: 2271-2285.

Elías Castillo, J., Ruiz Beltrán, L. (1979). Precipitaciones máximas en España. Ministerio de Agricultura, 545 pág, Madrid.

Gallart, F., Solé, A., Puigdefábregas, J., LÁZAro, R. (2002). Badland systems in the Mediterranean. En: Dryland rivers: Hydrology and Geomorphology of semi-arid channels (Bull, L. J., Kirkby, M. J., Eds). Wiley, pp. 299-326, Chichester.

García Fayos, P., Recatalà, Ma . T., Cerdà, A., Calvo, A. (1995). Seed population dynamics on badland slopes in SE Spain. Journal of Vegetation Science, 6: 691-696.

GArcía FAYOS, P., CERDÀ, A. (1997). Seed losses by surface wash in degraded Mediterranean environments. Catena, 29: 73-83.

García Fayos, P., García Ventoso, B., Cerdà, A. (2000). Limitations to plant establisment on eroded slopes in southeastern Spain. Journal of Vegetation Science, 1536: 77-86.

GArcía Ruiz, J.M., LASANTA, T., ALBERTO, F. (1997). Soil erosion by piping in irrigated fields. Geomorphology, 20: 269-278.

GERITS, J.J.P. (1991). Physico-chemical tresholds for sediment detachment, transport and deposition. Tesis doctoral inedita. Universiteit van Amsterdam, 186 pág., Amsterdam.

Gutiérrez Elorza, M., SAncho, C., Benito, G., Sirvent, J., Desir, G. (1997). Quantitative study of piping processes in badland areas of the Ebro basin, NE Spain. Geomorphology, 20: 237-253.

HARVEY, A.M. (1982). The role of piping in the development of badlands and gully systems in south-east Spain. En: Badland geomorphology and piping (Bryan, R., Yair, A., Eds.). University Press, pp. 317-336, Cambridge.

Harvey, A.M., CAlvo. A. (1991). Process interactions and rill development on badlands and gully slopes. Z. Geomorph. N. F. Suppl. Bd., 83: 175-94.

Harvey, A.M., CAlvo, A. (1991). Process interactions and rill development on badlands and gully slopes. Z. Geomorph. N. F. Suppl.-Bd., 83: 175-94. 
HILLS, R.C. (1970). The determination of the infiltration capacity on field soils using the cylinder infiltrometer. B.G.R.G., Technical bull., Geobooks Ltd., 24 pág., Norwich.

HodGES, W.K. (1982). Hydraulic characteristics of a badland pseudo-pediment slope system during simulated rain-storm experiments. En: Badland geomorphology and piping (Bryan, R., Yair, A., Eds.). University Press, pp. 127-152, Cambridge.

Hodges, W.K., Bryan, R.B. (1982). Runoff and Sediment Transport Dynamics in Canadian Badland Micro-Catchments. En: Badland geomorphology and piping (Bryan, R., Yair, A., Eds.). University Press, pp. 13-46, Cambridge.

HORTON, R.E. (1933). The role of infiltration in the hydrologic cicle. EOS Trans A.G.U., 14: 446-460.

HORTON, R.E. (1940). An approach toward a physical interpretation of infiltration capacity. Proc. Soil Sci. Soc. Am., 5: 399-417.

HowARD, A.D. (1994). Badlands. En: Geomorphology of Desert Environments (Abrahams, A.D., Parsons, A.J., Eds). pp. 213-242.

IMESON, A.C. (1983). Studies of erosion thresholds in semi-arid areas: field measurement of soil loss and infiltration in northern Morocco. Catena Suppl-Bd, 4: 79-89.

IMESON, A.C., KwAAD, F.J.P.M., Verstraten, J.M. (1982). The relationship of soil physical and chemical properties to the development of badlands in Morocco. En: Badland geomorphology and piping (Bryan, R., Yair, A., Eds.). University Press, pp. 47-70, Cambridge.

IMESON, A.C., VERSTRATEN. J.M. (1988). Rills on badland slopes: a physico-chemical controlled phenomenon. Catena suppl.-Bd., 12: 139-53.

JONES, J.A.A. (1981). The nature of soil piping - A review of research. BGRG Research Monograph, 301 pág.

LARONNE, J. (1982). Sediment and solute yield from Mancos Shale hillslopes. Colorado and Utah. En: Badland geomorphology and piping (Bryan, R., Yair, A., Eds.). University Press, pp. 181-194, Cambridge.

Leopold, L.B., Wolman, M.G., Miller, J.P. (1964). Fluvial Processes in Geomorphology, 522 pág., San Francisco.

LÓPEZ BERMÚdEZ, F. (1992). La erosión del suelo, un riesgo permanente de desertificación. Ecosistemas, 3: 10-13.

LÓPEZ BERMÚDEZ, F., TORCAL, L. (1986). Procesos de erosión en túnel (piping) en cuencas sedimentarias de Murcia (España). Estudio preliminar mediante difracción de rayos X y microscopio electrónico de barrido. Papeles de Geografía Física, 11: 7-20. 
LóPEZ BERMúdez, F., Romero DíAZ, M.A. (1989). Piping erosion and badland development in South-East Spain. Catena Suppl., 14: 59-73.

Meyer, A., Martínez Casasnovas, J.A. (1999). Prediction of existing gully erosion in vineyard parcels of the NE Spain: a logistic modelling approach. Soil and Tillage Research, 50: 319-331.

MEYER, L.D. (1994). Rainfall simulators for soil erosion research. En: Soil Erosion Research Methods (Lal, R., Ed.). Soil and Water Conservation Society (Ankeny), St. Lucie press, pp. 83-103, Delray Beach.

Nadal-Romero, E., RegüÉs, D., Martí Bono, C., Serrano Muela, P. (2006). Dinámica estacional de los procesos de meteorización en cárcavas del Pirineo Central. Rev. $C \&$ \& ., 20: 61-77.

Nadal Romero, E., Regués, D., Martí Bono, C., Serrano Muela, P. (2007). Badland dynamics in the Central Pyrenees: temporal and spatial patterns of weathering proceses. Earth Surface Processes and Landforms, 32: 888-904.

NiCOLAU, J.M. (2002). Runoff generation and routing on artificial slopes in a Mediterranean-continental environment: The Teruel coalfield, Spain. Hydrological Processes, 16: 631-647.

PAYÀ, J., CERDÀ, A. (1992). Cambios morfológicos y respuesta a la lluvia simulada de tres superficies de Badland. Petrer. Alacant. En: Estudios de Geomofología en Espa$\tilde{n} a$ (López Bermúdez, F., Conesa, C., Romero, M.A., Eds.). Sociedad Española de Geomorfología, pp. 161-170, Murcia.

Pérez Cueva, A. J. (1994). Atlas climático de la Comunidad Valenciana. Generalitat Valenciana, 243 pág., València.

RegüÉs, D., PARdini, G., PINI, R. (1992). Estudios de comportamientos de regolitos arcillosos frente a variaciones de la termperatura y la humedad. En: Estudios de Geomofología en España (López Bermúdez, F., Conesa, C., Romero, M.A., Eds.). Sociedad Española de Geomorfología, pp. 171-181, Murcia.

RegÜÉs, D., Llorens, P., PARdini, G., Pini, R.,Gallart, F. (1993). Physical weathering and regolith behaviour in a high erosion rate badland area at the Pyrenees: research design and first results. Pirineos, 141-142: 63-84.

REgÜÉS D., PARDINI G., GALlART F. (1995). Regolith behaviour and physical weathering of clayey mudrock as dependent on seasonal weather conditions in a badland area at Vallcebre, Eastern Pyrenees. Catena, 25: 199-212.

REGÜÉs, D., GALlART, F. (1996). Evolución de la respuesta hidrológica de un área arcillosa acarcavada, en relación con el estado físico superficial mediante experiencias de simulación de lluvia. Cadernos do Laboratorio Xeolóxico de Laxe, 21: 79-89. 
Regués, D., Gallart, F., Llorens, P., Latron, J., Soler, M. (1998). Las cuencas experimentales de Vallcebre: dinámica de sedimentos. En: Investigaciones recientes de la Geomorfología española (Gómez Ortiz A., Salvador, F., Eds.). Universidad de Barcelona, pp. 259-264, Barcelona.

REGÜÉS, D., GUÀRDIA, R., GALLART, F. (2000). Geomorphic agents versus vegetation spreading as causes of badland occurrence in a Mediterranean subhumid mountainous area. Catena, 40: 173-188.

REGÜÉS, D., GALLART, F. (2004). Seasonal patterns of runoff and erosion responses to simulated rainfall in a badland area in Mediterranean mountain conditions (Vallcebre, Southeastern Pyrenees). Earth Surface Processes and Landforms, 29: 755-767.

RodRíGueZ AizPEOLEA, J. (1992). Un ejemplo de la influencia del uso y abandono de bancales de fondo de canal en la evolución de Badlands (Petrer-Alacant). Actas del II Congreso Nacional de Geomorfología, pp. 211-220.

RÖMKENS, M.J.M., PRASAD, S.N., WHISLER, F.D. (1990). Surface sealing and infiltration. En: Process studies in hillslope hydrology (Anderson, M.G., Burt, T.P., Eds). pp. 127-172.

Schumm, A.S., Mosley, M.P., WeAVer, W.E. (1986). Experimental Fluvial Geomorpholgy. John Wiley and Sons, 413 pág., New York.

SCHUMm, S. A. (1964). Seasonal variations of erosion rates and processes on hillslopes in Western Colorado. Z. Geomorph. N.F., 5: 215-238.

SCHUMM, S.A., LuSBY, G.C. (1963). Seasonal variations of infiltration capacity and runoff on hillslopes in Western Colorado. Journal of Geophysical Research, 68: 3655-3666.

SCOGING, H. (1982). Spatial variations in infiltration runoff and erosion on hillslopes in semi-arid spain. En: Badland geomorphology and piping (Bryan, R., Yair, A., Eds.). University Press, pp. 80-112, Cambridge.

SCOGING, H.M., THORNES, J.B. (1980). Infiltration characteristics in a semiarid environment. IAHS Publication, 128: 159-168.

Sdao, G., Simone, A., VitTorini, S. (1984). Osservazioni geomorfolochique su calanchi e biancane in Calabria. Geogr.Fis. Diman. Quat.,10-16.

ShainberG, I., RoAdes, J.D., PRATHER, R.J. (1981). Effect of low electrolyte concentration on clay dispersion and hydraulic conductivity of a sodic soil. Soil Sci. Soc. Am. J., 45: 273-277.

Sirvent, J., Desir, G., Gutiérrez Elorza, M., SAnCHO, C., Benito, G. (1997). Erosion rates in badland areas recorded by collector, erosion pins and profilometer techniques (Ebro basin, NE-Spain). Geomorphology, 18: 61-75. 
SolÉ, A. (2006). Spain. En: Soil erosion in Europe (Boardman, J., Poesen, J., Eds.). Wiley, pp. 311-346, Chichester.

Solé, A., CAlvo, A., Cerdà, A., LÁZAro, R., Pini, R., BARbero, J. (1997). Influences of micro-relief patterns and plant cover on runoff related to processes in Badlands from Tabernas (SE Spain). Catena: 31, 28-38.

VITTORINI, S. (1977). Osservazioni sull'origine e sul ruolo di due forme di erosione nelle argile: calanchi e biancane. Boll. della Societa Geografica Italiana, VI: 25-54.

WELls, S.G., GuTIÉRREZ, A.A. (1982). Quaternary evolution of badlands in the southeastern Colorado Plateau, USA. En: Badland geomorphology and piping (Bryan, R., Yair, A., Eds.). University Press, pp. 239-258, Cambridge.

Wilcox, B.P., Wood, M.K., Tromble, J.T., WARD, T.J. (1986). A hand-portable single nozzle rainfall simulator designed for use on steep slopes. Journal of Range Management, 39: 375-377.

Wise, S.M., ThORnes, J.B., Gilman, A. (1982). How old are the badlands?. A case study from south-east Spain. En: Badland geomorphology and piping (Bryan, R., Yair, A., Eds.). University Press, pp. 259-278, Cambridge.

WoOD, M.K. (1987). Plot numbers required to determine infiltration rates and sediment production on rangelands in south central New Mexico. Journal of Range Management, 40: 259-63.

YAIR, A., LAVEE, H., BRYAN, R.B., ADAR, E. (1980). Runoff and erosion processes and rates in the Zin Valley Badlands, northern Negev, Israel. Earth Surface Processes and Landforms, 5: 205-225.

YAIR, A., Goldberg, P., Brimer, B. (1982). Long term denudation rates in the ZinHavarim badlands, northern Negev, Israel. Badland geomorphology and piping (Bryan, R., Yair, A., Eds.). University Press, pp. 279-292, Cambridge. 